Supplement of Biogeosciences, 15, 3475-3496, 2018

https://doi.org/10.5194/bg-15-3475-2018-supplement

(C) Author(s) 2018. This work is distributed under

the Creative Commons Attribution 4.0 License.

(c) (1)

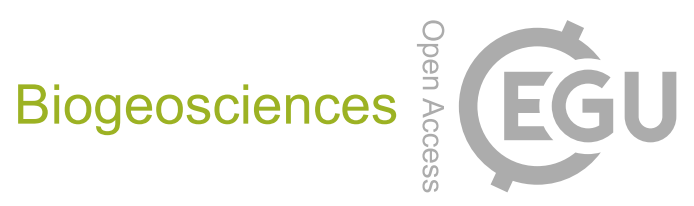

Supplement of

\title{
The influence of soil properties and nutrients on conifer forest growth in Sweden, and the first steps in developing a nutrient availability metric
}

\section{Kevin Van Sundert et al.}

Correspondence to: Kevin Van Sundert (kevin.vansundert@uantwerpen.be)

The copyright of individual parts of the supplement might differ from the CC BY 4.0 License. 


\section{The productivity vs climate regression model}

We disentangled the influence of climatological variables (TSUM and precipitation) on productivities of spruce and pine forests using ANCOVA (Analysis of Covariance). All possible combinations of single continuous variables and their interactions with species were alternatively included in data-driven regression models to finally select the one with the

5 lowest mean square of prediction error according to 10-fold cross-validation (package DAAG - Maindonald and Braun, 2015). In addition, potential non-linearities were detected with a generalized additive model from the mgcv package (Wood, 2006) and histograms of the variables. Assumptions of linearity, normality of residuals, homoscedasticity, outliers and noncollinearity were tested with the methods mentioned in the main text.

$\operatorname{MAI}\left[\mathrm{m}^{3} \mathrm{ha}^{-1} \mathrm{yr}^{-1}\right]=\mathrm{ax} \operatorname{TSUM}^{2}\left[{ }^{\circ} \mathrm{C}\right.$ days $]+\mathrm{b}$ x TSUM $\left[{ }^{\circ} \mathrm{C}\right.$ days $]+\mathrm{cx} \operatorname{MAP}[\mathrm{mm}]+\mathrm{d}$,

10 
45 Table S1. Overall mean squared error (ms) after 10-fold cross-validation for the most relevant (i.e. TSUM and spp clearly had to be included) candidate model structures that explain variation in productivity (mean annual increment $-\mathrm{MAI}-\mathrm{m}^{3} \mathrm{ha}^{-1} \mathrm{yr}^{-1}$ ) by climate (TSUM and MAP) and species across Swedish spruce and pine forests. The selected model is marked in gray. Abbreviations: TSUM = growing season temperature sum $\left[{ }^{\circ} \mathrm{C}\right.$ days]; MAP $=$ mean annual precipitation $[\mathrm{mm}]$; $\mathrm{spp}=$ species.

\begin{tabular}{|c|c|c|c|}
\hline Variables in model & $\begin{array}{c}\text { Overall } \\
\text { ms }\end{array}$ & Variables in model & $\begin{array}{c}\text { Overal } \\
\text { ms }\end{array}$ \\
\hline TSUM²:spp, TSUM:spp, TSUM ${ }^{2}, T S U M, M A P, s p p$ & 1.48 & TSUM:spp, MAP, TSUM, spp & 1.63 \\
\hline $\begin{array}{l}\text { TSUM } 2 \text { :spp, TSUM:spp, MAP:spp, TSUM }{ }^{2} \text {, TSUM, } \\
\text { MAP, spp }\end{array}$ & 1.48 & TSUM:spp, TSUM, spp & 1.64 \\
\hline TSUM $^{2}:$ spp, TSUM:spp, TSUM, spp & 1.49 & TSUM $^{2}$, TSUM, MAP, spp & 2.23 \\
\hline TSUM², TSUM:spp, MAP:spp, TSUM, MAP, spp & 1.58 & TSUM $^{2}$, TSUM, spp & 2.24 \\
\hline TSUM:spp, TSUM², TSUM, MAP, spp & 1.59 & MAP:spp, TSUM, MAP, spp & 2.24 \\
\hline TSUM:spp, TSUM², TSUM, spp & 1.60 & TSUM, MAP, spp & 2.27 \\
\hline TSUM:spp, MAP:spp, TSUM, MAP, spp & 1.61 & TSUM, spp & 2.28 \\
\hline
\end{tabular}

50

55

60

65 
85 Table S2. Species specific estimates, statistics ( $t$ and partial $\left.R^{2}\right)$ and significance ( $P$ values) for the parameters in Eq. $(\mathrm{S} 1)$ and $F$ statistics and significance for species differences in these estimates. (Partial) $R^{2}$ values were approximated based on comparing fitted values with actual productivities.

\begin{tabular}{|c|c|c|c|c|c|c|c|}
\hline \multirow[t]{2}{*}{ Parameter } & \multirow{2}{*}{$\begin{array}{c}\text { Species } \\
\text { difference }\end{array}$} & \multicolumn{3}{|c|}{ Spruce } & \multicolumn{3}{|c|}{ Pine } \\
\hline & & Estimate & Stat & & Estimate & Stati & tics \\
\hline $\mathrm{a}$ & $\begin{array}{l}F_{1,2475}=175.3 \\
P<0.01\end{array}$ & $\begin{array}{l}(9.0 \pm 0.6) \\
\quad \times 10^{-6}\end{array}$ & $\begin{array}{l}t_{1071}=14.15 \\
P<0.01\end{array}$ & & $\begin{array}{l}(-3.0 \pm 0.6) \\
\quad \times 10^{-6}\end{array}$ & $\begin{array}{l}t_{1403}=-4.64 \\
P<0.01\end{array}$ & \\
\hline $\mathrm{b}$ & $\begin{array}{l}F_{1,2475} \\
\quad=1112.2 \\
P<0.01\end{array}$ & $\begin{array}{l}-0.008 \\
\pm 0.001\end{array}$ & $\begin{array}{l}t_{1071}=-5.76 \\
P<0.01\end{array}$ & $R^{2} \approx 0.854$ & $\begin{array}{l}0.011 \\
\quad \pm 0.001\end{array}$ & $\begin{array}{l}t_{1403}=7.80 \\
P<0.01\end{array}$ & $R^{2} \approx 0.480$ \\
\hline $\mathrm{c}$ & $\mathrm{N} / \mathrm{A}^{\mathrm{a}}$ & $\begin{array}{l}(0.3 \pm 0.3) \\
\quad \times 10^{-3}\end{array}$ & $\begin{array}{l}t_{1071}=0.97 \\
P=0.33\end{array}$ & $R^{2} \approx 0.001$ & $\begin{array}{l}(0.3 \pm 0.3) \\
\quad \mathrm{x} 10^{-3}\end{array}$ & $\begin{array}{l}t_{1403}=0.97 \\
P=0.33\end{array}$ & $R^{2} \approx 0$ \\
\hline d & $\begin{array}{l}F_{1,2475} \\
\quad=1731.1 \\
P<0.01\end{array}$ & $3.5 \pm 0.8$ & $\begin{array}{l}t_{1071}=4.59 \\
P<0.01\end{array}$ & & $-4.1 \pm 0.7$ & $\begin{array}{l}t_{1403}=-5.24 \\
P<0.01\end{array}$ & \\
\hline Total & & & & $R^{2} \approx 0.856$ & $R^{2}=0.805$ & & $R^{2} \approx 0.480$ \\
\hline
\end{tabular}

${ }^{\mathrm{a} A}$ regression model without MAP $*$ species interaction was selected by the cross-validation procedure (Table S1). 
90 Table S3. Association between ( $\log$ ) N deposition and normalized productivity, stratified by soil moisture class (dry to moist). Significance ( $P$-values) of $\mathrm{N}$ deposition on residual productivity (mean annual increment - MAI $\left[\mathrm{m}^{3} \mathrm{ha}^{-1} \mathrm{yr}^{-1}\right]$ ) and actual/attainable MAI (for spruce only) across Sweden are given. For (near) significant variables (i.e. $P<0.10$ ), parameter estimates \pm s.e.m. and the proportion

\begin{tabular}{|c|c|c|c|c|c|}
\hline \multirow{2}{*}{$\begin{array}{l}\text { Normalized } \\
\text { productivity } \\
\text { response }\end{array}$} & \multirow[t]{2}{*}{ Region } & \multicolumn{4}{|l|}{ 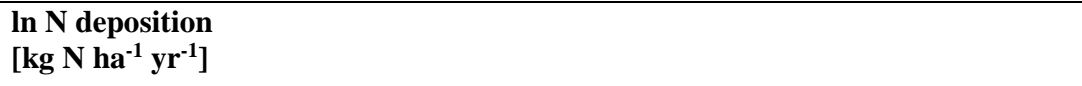 } \\
\hline & & Dry & Fresh & Fresh-moist & Moist \\
\hline \multirow[t]{3}{*}{$\begin{array}{l}\text { Residual MAI } \\
(\operatorname{method} 1)\end{array}$} & $\mathrm{N}$ & $\begin{array}{l}\text { slope }=0.7 \pm 0.3 \\
P=0.04 \\
\text { intercept } \\
\quad=-1.0 \pm 0.2 \\
P<0.01 \\
R^{2}=0.100 \\
n=32\end{array}$ & $\begin{array}{l}P=0.51 \\
n=399\end{array}$ & $\begin{array}{l}P=0.37 \\
n=132\end{array}$ & $\begin{array}{l}P=0.26 \\
n=5\end{array}$ \\
\hline & M & $\begin{array}{l}P=0.63 \\
n=50\end{array}$ & $\begin{array}{l}\text { slope }=0.6 \pm 0.2 \\
P<0.01 \\
\text { intercept } \\
\quad=-0.2 \pm 0.1 \\
P<0.01 \\
R^{2}=0.022 \\
n=549\end{array}$ & $\begin{array}{l}P=0.99 \\
n=237\end{array}$ & $\begin{array}{l}P=0.29 \\
n=7\end{array}$ \\
\hline & S & $\begin{array}{l}P=0.79 \\
n=62\end{array}$ & $\begin{array}{l}P=0.39 \\
n=742\end{array}$ & $\begin{array}{l}P=0.42 \\
n=233\end{array}$ & $\begin{array}{l}\text { slope }=-3 \pm 1 \\
P=0.04 \\
\text { intercept }=3 \pm 2 \\
P=0.23 \\
R^{2}=0.201 \\
n=17\end{array}$ \\
\hline $\begin{array}{l}\text { Actual/attainable } \\
\text { MAI } \\
(\text { method } 2)\end{array}$ & $\begin{array}{l}\text { entire } \\
\text { Sweden }\end{array}$ & $\begin{array}{l}\text { slope }=19 \pm 5 \\
P<0.01 \\
\text { intercept }=8 \pm 9 \\
P=0.36 \\
R^{2}=0.373 \\
n=22\end{array}$ & $\begin{array}{l}\text { slope }=14.6 \pm 0.5 \\
P<0.01 \\
\text { intercept } \\
\quad=22.7 \pm 0.8 \\
P<0.01 \\
R^{2}=0.565 \\
n=702\end{array}$ & $\begin{array}{l}\text { slope }=12.7 \pm 0.8 \\
P<0.01 \\
\text { intercept } \\
\quad=20 \pm 1 \\
P<0.01 \\
R^{2}=0.415 \\
n=318\end{array}$ & $\begin{array}{l}\text { slope }=12 \pm 4 \\
P=0.02 \\
\text { intercept } \\
\quad=21 \pm 4 \\
P<0.01 \\
R^{2}=0.384 \\
n=11\end{array}$ \\
\hline
\end{tabular}

of variation explained $\left(R^{2}\right)$ are shown as well. Abbreviations: $\mathrm{N}=$ north; $\mathrm{M}=$ middle; $\mathrm{S}=$ south. 
Table S4. Association between ( $\log ) \mathrm{N}$ deposition and normalized productivity, stratified by soil type. Significance $(P$-values) of $\mathrm{N}$ deposition on residual productivity (mean annual increment - MAI $\left[\mathrm{m}^{3} \mathrm{ha}^{-1} \mathrm{yr}^{-1}\right]$ ) and actual/attainable MAI (for spruce only) across Sweden are given. For (near) significant variables (i.e. $P<0.10$ ), parameter estimates \pm s.e.m. and the proportion of variation explained $\left(R^{2}\right)$ are shown as well. Abbreviations: $\mathrm{N}=$ north; $\mathrm{M}=$ middle; $\mathrm{S}=$ south.

\begin{tabular}{|c|c|c|c|c|c|c|}
\hline \multirow{2}{*}{$\begin{array}{l}\text { Normalized } \\
\text { productivity } \\
\text { response }\end{array}$} & \multirow[t]{2}{*}{ Region } & \multicolumn{5}{|l|}{$\begin{array}{l}\text { In } \mathrm{N} \text { deposition } \\
{\left[\mathrm{kg} \mathrm{N} \mathrm{ha}^{-1} \mathrm{yr}^{-1}\right]}\end{array}$} \\
\hline & & Histosol & Gleysol & Regosol & Leptosol & Podzol \\
\hline \multirow[t]{3}{*}{$\begin{array}{l}\text { Residual MAI } \\
\text { (method 1) }\end{array}$} & $\mathrm{N}$ & $\begin{array}{l}P=0.13 \\
n=13\end{array}$ & $\begin{array}{l}P=0.23 \\
n=30\end{array}$ & $\begin{array}{l}P=0.86 \\
n=217\end{array}$ & $\begin{array}{l}P=0.87 \\
n=23\end{array}$ & $\begin{array}{l}P=0.57 \\
n=268\end{array}$ \\
\hline & M & $\begin{array}{l}\text { slope }=0.8 \pm 0.4 \\
P=0.09 \\
\text { intercept } \\
\quad=-2.0 \pm 0.5 \\
P<0.01 \\
R^{2}=0.031 \\
n=60\end{array}$ & $\begin{array}{l}P=0.66 \\
n=28\end{array}$ & $\begin{array}{l}P=0.60 \\
n=277\end{array}$ & $\begin{array}{l}P=0.25 \\
n=41\end{array}$ & $\begin{array}{l}\text { slope }=1.0 \pm 0.2 \\
P<0.01 \\
\text { intercept } \\
\quad=-0.8 \pm 0.2 \\
P<0.01 \\
R^{2}=0.064 \\
n=400\end{array}$ \\
\hline & S & $\begin{array}{l}P=0.77 \\
n=101\end{array}$ & $\begin{array}{l}P=0.51 \\
n=37\end{array}$ & $\begin{array}{l}P=0.44 \\
n=372\end{array}$ & $\begin{array}{l}P=0.64 \\
n=116\end{array}$ & $\begin{array}{l}P=0.70 \\
n=324\end{array}$ \\
\hline $\begin{array}{l}\text { Actual/attainable } \\
\text { MAI } \\
(\operatorname{method} 2)\end{array}$ & $\begin{array}{l}\text { entire } \\
\text { Sweden }\end{array}$ & $\begin{array}{l}\text { slope }=19 \pm 2 \\
P<0.01 \\
\text { intercept }=10 \pm 3 \\
P<0.01 \\
R^{2}=0.541 \\
n=64\end{array}$ & $\begin{array}{l}\text { slope }=10 \pm 2 \\
P<0.01 \\
\text { intercept } \\
\quad=26 \pm 2 \\
P<0.01 \\
R^{2}=0.404 \\
n=63\end{array}$ & $\begin{array}{l}\text { slope }=15.5 \pm 0.7 \\
P<0.01 \\
\text { intercept } \\
\quad=21 \pm 1 \\
P<0.01 \\
R^{2}=0.578 \\
n=377\end{array}$ & $\begin{array}{l}\text { slope }=12 \pm 2 \\
P<0.01 \\
\text { intercept } \\
\quad=22 \pm 4 \\
P<0.01 \\
R^{2}=0.270 \\
n=70\end{array}$ & $\begin{array}{l}\text { slope }=14.3 \pm 0.6 \\
P<0.01 \\
\text { intercept } \\
\quad=21.2 \pm 0.9 \\
P<0.01 \\
R^{2}=0.581 \\
n=399\end{array}$ \\
\hline
\end{tabular}

110 
Table S5. Associations between single soil variables and normalized productivity of Table 2, stratified by soil moisture class (dry to moist). Significance ( $P$-values) of single soil variable effects on residual productivity (mean annual increment - MAI $\left[\mathrm{m}^{3} \mathrm{ha}^{-1} \mathrm{yr}^{-1}\right]$ ) and actual/attainable MAI (for spruce only) across Sweden are given. For (near) significant variables (i.e. $P<0.10$ ), parameter estimates \pm s.e.m. and the proportion of variation explained $\left(R^{2}\right)$ are shown as well. Abbreviations: $\mathrm{N}=$ north; $\mathrm{M}=$ middle; $\mathrm{S}=$ south; $\mathrm{SOC}=$ soil organic carbon concentration; $\mathrm{Soil} \mathrm{C}: \mathrm{N}=$ soil carbon to nitrogen ratio; $\mathrm{TEB}=$ total exchangeable bases; quad = parameter estimate for quadratic term; lin = parameter estimate for linear term of a quadratic function.

\begin{tabular}{|c|c|c|c|c|c|}
\hline \multirow[t]{2}{*}{$\begin{array}{l}\text { Normalized } \\
\text { productivity } \\
\text { response }\end{array}$} & \multirow[t]{2}{*}{ Region } & \multirow{2}{*}{$\begin{array}{l}\text { In SOC } \\
0-20 \mathrm{~cm} \\
{[\%]} \\
\text { Dry }\end{array}$} & \multirow[b]{2}{*}{ Fresh } & \multirow[b]{2}{*}{ Fresh-moist } & \multirow[b]{2}{*}{ Moist } \\
\hline & & & & & \\
\hline \multirow[t]{3}{*}{$\begin{array}{l}\text { Residual MAI } \\
\text { (method 1) }\end{array}$} & $\mathrm{N}$ & $\begin{array}{l}P=0.32 \\
n=32\end{array}$ & $\begin{array}{l}\text { quad }=-0.13 \pm 0.02 \\
P<0.01 \\
\text { lin }=0.39 \pm 0.10 \\
P<0.01 \\
\text { intercept } \\
\quad=-0.05 \pm 0.10 \\
R^{2} \text { tot }=0.052 \\
n_{\text {tot }}=1569\end{array}$ & $\begin{array}{l}\text { quad }=-0.22 \pm 0.06 \\
P<0.01 \\
\text { lin }=0.9 \pm 0.3 \\
P<0.01 \\
\text { intercept }=-0.8 \pm 0.3 \\
P=0.01 \\
R^{2}{ }_{t o t}=0.106 \\
n_{t o t}=830\end{array}$ & $\begin{array}{l}\text { quad }=-3 \pm 1 \\
P=0.03 \\
\text { lin }=15 \pm 7 \\
P=0.03 \\
\text { intercept }=-23 \pm 10 \\
P=0.03 \\
R^{2} \text { tot }=0.314 \\
n_{\text {tot }}=4\end{array}$ \\
\hline & M & $\begin{array}{l}P=0.75 \\
n=47\end{array}$ & $\begin{array}{l}\text { quad }=-0.13 \pm 0.02 \\
P<0.01 \\
\text { lin }=0.33 \pm 0.09 \\
P<0.01 \\
\text { intercept } \\
\quad=0.17 \pm 0.09 \\
P=0.06 \\
R_{\text {tot }}^{2}=0.052 \\
n_{\text {tot }}=1569\end{array}$ & $\begin{array}{l}\text { quad }=-0.22 \pm 0.06 \\
P<0.01 \\
\text { lin }=0.8 \pm 0.1 \\
P<0.01 \\
\text { intercept }=-0.9 \pm 0.3 \\
P<0.01 \\
R^{2} \text { tot }=0.106 \\
n_{t o t}=830\end{array}$ & $\begin{array}{l}\text { quad }=-3 \pm 1 \\
P=0.03 \\
\text { lin }=13 \pm 5 \\
P=0.03 \\
\text { intercept }=-14 \pm 6 \\
P=0.02 \\
R_{\text {tot }}^{2}=0.314 \\
n_{\text {tot }}=7\end{array}$ \\
\hline & S & $\begin{array}{l}P=0.10 \\
n=44\end{array}$ & $\begin{array}{l}\text { quad }=-0.13 \pm 0.02 \\
P<0.01 \\
\text { slope }=0.2 \pm 0.1 \\
P=0.09 \\
\text { intercept }=0.5 \pm 0.1 \\
P<0.01 \\
R^{2}{ }_{t o t}=0.052 \\
n_{\text {tot }}=1569\end{array}$ & $\begin{array}{l}\text { quad }=-0.22 \pm 0.06 \\
P<0.01 \\
\text { lin }=0.7 \pm 0.3 \\
P=0.03 \\
\text { intercept }=-0.6 \pm 0.4 \\
P=0.19 \\
R_{\text {tot }}^{2}=0.106 \\
n_{\text {tot }}=830\end{array}$ & $\begin{array}{l}\text { quad }=-3 \pm 1 \\
P<0.01 \\
\text { lin }=13 \pm 6 \\
P=0.05 \\
\text { intercept }=-16 \pm 9 \\
P=0.07 \\
R^{2} \text { tot }=0.314 \\
n_{\text {tot }}=17\end{array}$ \\
\hline $\begin{array}{l}\text { Actual/attainable } \\
\text { MAI } \\
\text { (method } 2)\end{array}$ & $\begin{array}{l}\text { entire } \\
\text { Sweden }\end{array}$ & $\begin{array}{l}P=0.66 \\
n=19\end{array}$ & $\begin{array}{l}\text { quad }=-1.6 \pm 0.5 \\
P<0.01 \\
\text { lin }=9 \pm 2 \\
P<0.01 \\
\text { intercept } \\
\quad=34 \pm 2 \\
P<0.01 \\
R^{2}=0.043 \\
n=641\end{array}$ & $\begin{array}{l}\text { quad }=-3.4 \pm 0.8 \\
P<0.01 \\
\text { lin }=16 \pm 4 \\
P<0.01 \\
\text { intercept } \\
\quad=19 \pm 4 \\
P<0.01 \\
R^{2}=0.056 \\
n=285\end{array}$ & $\begin{array}{l}P=0.53 \\
n=10\end{array}$ \\
\hline
\end{tabular}


Table S5 (continued).

\begin{tabular}{|c|c|c|c|c|c|}
\hline \multirow[t]{2}{*}{$\begin{array}{l}\text { Normalized } \\
\text { productivity } \\
\text { response }\end{array}$} & \multirow[t]{2}{*}{ Region } & \multirow{2}{*}{$\begin{array}{l}\text { In N stock } \\
0-20 \mathrm{~cm}^{-2} \\
{\left[\mathrm{~g} \mathrm{~m}^{-2}\right]} \\
\text { Dry } \\
\end{array}$} & \multirow[b]{2}{*}{ Fresh } & \multirow[b]{2}{*}{ Fresh-moist } & \multirow[b]{2}{*}{ Moist } \\
\hline & & & & & \\
\hline \multirow[t]{3}{*}{$\begin{array}{l}\text { Residual MAI } \\
\text { (method 1) }\end{array}$} & $\mathrm{N}$ & $\begin{array}{l}P=0.56 \\
n_{\text {tot }}=123\end{array}$ & $\begin{array}{l}\text { slope }=0.22 \pm 0.06 \\
P<0.01 \\
\text { intercept }=-1.0 \pm 0.3 \\
P<0.01 \\
R^{2}{ }_{t o t}=0.007 \\
n_{t o t}=1569\end{array}$ & $\begin{array}{l}\text { slope } \\
\quad=0.26 \pm 0.08 \\
P<0.01 \\
\text { intercept } \\
\quad=-1.5 \pm 0.4 \\
P<0.01 \\
R^{2}{ }_{t o t}=0.012 \\
n_{\text {tot }}=830\end{array}$ & $\begin{array}{l}P=0.78 \\
n_{\text {tot }}=28\end{array}$ \\
\hline & M & $\begin{array}{l}P=0.56 \\
n_{\text {tot }}=123\end{array}$ & $\begin{array}{l}\text { slope }=0.22 \pm 0.06 \\
P<0.01 \\
\text { intercept }=-1.0 \pm 0.3 \\
P<0.01 \\
R^{2}{ }_{t o t}=0.007 \\
n_{\text {tot }}=1569\end{array}$ & $\begin{array}{l}\text { slope }=0.26 \pm 0.08 \\
P<0.01 \\
\text { intercept } \\
\quad=-1.5 \pm 0.4 \\
P<0.01 \\
R_{\text {tot }}^{2}=0.012 \\
n_{t o t}=830\end{array}$ & $\begin{array}{l}P=0.78 \\
n_{\text {tot }}=28\end{array}$ \\
\hline & S & $\begin{array}{l}P=0.56 \\
n_{t o t}=123\end{array}$ & $\begin{array}{l}\text { slope }=0.22 \pm 0.06 \\
P<0.01 \\
\text { intercept }=-1.0 \pm 0.3 \\
P<0.01 \\
R^{2}{ }_{t o t}=0.007 \\
n_{\text {tot }}=1569\end{array}$ & $\begin{array}{l}\text { slope }=0.26 \pm 0.08 \\
P<0.01 \\
\text { intercept } \\
\quad=-1.5 \pm 0.4 \\
P<0.01 \\
R^{2}{ }_{t o t}=0.012 \\
n_{t o t}=830\end{array}$ & $\begin{array}{l}P=0.78 \\
n_{t o t}=28\end{array}$ \\
\hline $\begin{array}{l}\text { Actual/attainable } \\
\text { MAI } \\
\text { (method 2) }\end{array}$ & $\begin{array}{l}\text { entire } \\
\text { Sweden }\end{array}$ & $\begin{array}{l}P=0.10 \\
n=19\end{array}$ & $\begin{array}{l}\text { slope }=11 \pm 1 \\
P<0.01 \\
\text { intercept } \\
\quad=-19 \pm 6 \\
P<0.01 \\
R^{2}=0.149 \\
n=641\end{array}$ & $\begin{array}{l}\text { slope }=10 \pm 1 \\
P<0.01 \\
\text { intercept } \\
\quad=-2 \pm 7 \\
P<0.01 \\
R^{2}=0.212 \\
n=285\end{array}$ & $\begin{array}{l}P=0.12 \\
n=10\end{array}$ \\
\hline
\end{tabular}


Table S5 (continued).

\begin{tabular}{|c|c|c|c|c|c|}
\hline \multirow{2}{*}{$\begin{array}{l}\text { Normalized } \\
\text { productivity } \\
\text { response }\end{array}$} & \multirow[t]{2}{*}{ Region } & \multicolumn{4}{|l|}{$\begin{array}{l}\text { Soil C:N } \\
0-20 \mathrm{~cm}\end{array}$} \\
\hline & & Dry & Fresh & Fresh-moist & Moist \\
\hline \multirow[t]{3}{*}{$\begin{array}{l}\text { Residual MAI } \\
(\operatorname{method} 1)\end{array}$} & $\mathrm{N}$ & $\begin{array}{l}P=0.20 \\
n=32\end{array}$ & $\begin{array}{l}\text { slope } \\
\quad=-0.007 \pm 0.004 \\
P=0.09 \\
\text { intercept }=0.3 \pm 0.1 \\
P<0.01 \\
R^{2}=0.005 \\
n=385\end{array}$ & $\begin{array}{l}\text { slope } \\
\quad=-0.040 \pm 0.008 \\
P<0.01 \\
\text { intercept }=0.8 \pm 0.2 \\
P<0.01 \\
R^{2}=0.184 \\
n=121\end{array}$ & $\begin{array}{l}P=0.13 \\
n=4\end{array}$ \\
\hline & M & $\begin{array}{l}P=0.22 \\
n=47\end{array}$ & $\begin{array}{l}\text { slope } \\
\quad=-0.015 \pm 0.006 \\
P=0.02 \\
\text { intercept }=0.7 \pm 0.2 \\
P<0.01 \\
R^{2}=0.008 \\
n=515\end{array}$ & $\begin{array}{l}\text { slope } \\
\quad=-0.039 \pm 0.010 \\
P<0.01 \\
\text { intercept }=0.5 \pm 0.3 \\
P=0.09 \\
R^{2}=0.067 \\
n=208\end{array}$ & $\begin{array}{l}P=0.39 \\
n=7\end{array}$ \\
\hline & $\mathrm{S}$ & $\begin{array}{l}P=0.28 \\
n=44\end{array}$ & $\begin{array}{l}\text { slope } \\
\quad=-0.048 \pm 0.009 \\
P<0.01 \\
\text { intercept }=1.5 \pm 0.2 \\
P<0.01 \\
R^{2}=0.041 \\
n=669\end{array}$ & $\begin{array}{l}\text { slope } \\
\quad=-0.09 \pm 0.01 \\
P<0.01 \\
\text { intercept }=1.8 \pm 0.4 \\
P<0.01 \\
R^{2}=0.170 \\
n=216\end{array}$ & $\begin{array}{l}P=0.14 \\
n=17\end{array}$ \\
\hline $\begin{array}{l}\text { Actual/attainable } \\
\text { MAI } \\
\text { (method 2) }\end{array}$ & $\begin{array}{l}\text { entire } \\
\text { Sweden }\end{array}$ & N/A & N/A & N/A & N/A \\
\hline
\end{tabular}


Table S5 (continued).

\begin{tabular}{llllll}
\hline $\begin{array}{l}\text { Normalized } \\
\text { productivity } \\
\text { response }\end{array}$ & Region & $\begin{array}{l}\text { In soil C:N } \\
\mathbf{0 - 1 0} \mathbf{c m}\end{array}$ & & & \\
& & & & & \\
& & Dry & Fresh & Fresh-moist & Moist \\
\hline $\begin{array}{llll}\text { Residual MAI } \\
\text { (method 1) }\end{array}$ & $\mathrm{N}$ & N/A & N/A & N/A & N/A \\
& M & N/A & N/A & N/A & N/A \\
Actual/attainable & N & N/A & N/A & N/A & N/A \\
MAI & slope & slope $=-17 \pm 2$ & slope $=-19 \pm 3$ & $P=0.35$ \\
(method 2) & Sweden & $=-33 \pm 15$ & $P<0.01$ & $P<0.01$ & $n=10$ \\
& & $P=0.04$ & intercept $=96 \pm 6$ & intercept $=95 \pm 8$ & \\
& & intercept & $P<0.01$ & $P<0.01$ & \\
& $=143 \pm 47$ & $R^{2}=0.112$ & $R^{2}=0.149$ & \\
& & $P<0.01$ & $n=641$ & $n=285$ & \\
& & $R^{2}=0.174$ & & & \\
& $n=19$ & & & \\
\hline
\end{tabular}


Table S5 (continued).

\begin{tabular}{|c|c|c|c|c|c|}
\hline \multirow{2}{*}{$\begin{array}{l}\text { Normalized } \\
\text { productivity } \\
\text { response }\end{array}$} & \multirow[t]{2}{*}{ Region } & \multicolumn{4}{|l|}{$\begin{array}{l}\text { Mineral soil sand } \\
{[\%]}\end{array}$} \\
\hline & & Dry & Fresh & Fresh-moist & Moist \\
\hline \multirow[t]{3}{*}{$\begin{array}{l}\text { Residual MAI } \\
(\text { method } 1)\end{array}$} & $\mathrm{N}$ & $\begin{array}{l}\text { slope } \\
\quad=0.021 \pm 0.009 \\
P=0.02 \\
\text { intercept } \\
\quad=-1.3 \pm 0.8 \\
P=0.10 \\
R_{\text {tot }}^{2}=0.101 \\
n_{t o t}=123\end{array}$ & $\begin{array}{l}\text { slope }=0.004 \pm 0.001 \\
P<0.01 \\
\text { intercept }=-0.2 \pm 0.1 \\
P=0.04 \\
R_{\text {tot }}^{2}=0.006 \\
n_{t o t}=1569\end{array}$ & $\begin{array}{l}\text { slope } \\
\quad=0.006 \pm 0.002 \\
P<0.01 \\
\text { intercept } \\
\quad=-0.4 \pm 0.1 \\
P<0.01 \\
R^{2} \text { tot }=0.015 \\
n_{\text {tot }}=830\end{array}$ & $\begin{array}{l}P=0.64 \\
n_{\text {tot }}=28\end{array}$ \\
\hline & M & $\begin{array}{l}\text { slope } \\
\quad=0.021 \pm 0.009 \\
P=0.02 \\
\text { intercept } \\
\quad=-1.0 \pm 0.6 \\
P=0.09 \\
R_{\text {tot }}^{2}=0.101 \\
n_{\text {tot }}=123\end{array}$ & $\begin{array}{l}\text { slope }=0.004 \pm 0.001 \\
P<0.01 \\
\text { intercept } \\
\quad=-0.15 \pm 0.09 \\
P=0.10 \\
R^{2}{ }_{t o t}=0.006 \\
n_{t o t}=1569\end{array}$ & $\begin{array}{l}\text { slope } \\
\quad=0.006 \pm 0.002 \\
P<0.01 \\
\text { intercept } \\
\quad=-0.6 \pm 0.1 \\
P<0.01 \\
R^{2}{ }_{t o t}=0.015 \\
n_{t o t}=830\end{array}$ & $\begin{array}{l}P=0.64 \\
n_{t o t}=28\end{array}$ \\
\hline & S & $\begin{array}{l}\text { slope } \\
\quad=0.021 \pm 0.009 \\
P=0.02 \\
\text { intercept } \\
\quad=-1.6 \pm 0.5 \\
P<0.01 \\
R^{2}{ }_{t o t}=0.101 \\
n_{t o t}=123\end{array}$ & $\begin{array}{l}\text { slope }=0.004 \pm 0.001 \\
P<0.01 \\
\text { intercept } \\
\quad=-0.01 \pm 0.08 \\
P=0.86 \\
R^{2}{ }_{t o t}=0.006 \\
n_{\text {tot }}=1569\end{array}$ & $\begin{array}{l}\text { slope } \\
\quad=0.006 \pm 0.002 \\
P<0.01 \\
\text { intercept } \\
\quad=-0.5 \pm 0.1 \\
P<0.01 \\
R^{2}{ }_{t o t}=0.015 \\
n_{\text {tot }}=830\end{array}$ & $\begin{array}{l}P=0.64 \\
n_{\text {tot }}=28\end{array}$ \\
\hline
\end{tabular}


Table S5 (continued).

\begin{tabular}{|c|c|c|c|c|c|}
\hline \multirow{2}{*}{$\begin{array}{l}\text { Normalized } \\
\text { productivity } \\
\text { response }\end{array}$} & \multirow[t]{2}{*}{ Region } & \multicolumn{4}{|l|}{$\begin{array}{l}\text { Mineral soil clay } \\
{[\%]}\end{array}$} \\
\hline & & Dry & Fresh & Fresh-moist & Moist \\
\hline \multirow[t]{3}{*}{$\begin{array}{l}\text { Residual MAI } \\
\text { (method 1) }\end{array}$} & $\mathrm{N}$ & $\begin{array}{l}\text { slope }=0.10 \pm 0.05 \\
P=0.03 \\
\text { intercept } \\
\quad=-0.8 \pm 0.3 \\
P=0.02 \\
R^{2} \text { tot }=0.072 \\
n_{\text {tot }}=123\end{array}$ & $\begin{array}{l}\text { slope }=0.011 \pm 0.005 \\
P=0.02 \\
\text { intercept } \\
\quad=0.06 \pm 0.04 \\
P=0.14 \\
R^{2} \text { tot }=0.003 \\
n_{t o t}=1569\end{array}$ & $\begin{array}{l}P=0.97 \\
n_{\text {tot }}=545\end{array}$ & $\begin{array}{l}\text { slope }=0.05 \pm 0.02 \\
P=0.03 \\
\text { intercept } \\
\quad=-0.6 \pm 0.2 \\
P=0.03 \\
R^{2}{ }_{\text {tot }}=0.124 \\
n_{\text {tot }}=28\end{array}$ \\
\hline & M & $\begin{array}{l}\text { slope }=0.10 \pm 0.05 \\
P=0.03 \\
\text { intercept } \\
\quad=-0.8 \pm 0.3 \\
P=0.02 \\
R_{\text {tot }}^{2}=0.072 \\
n_{t o t}=123\end{array}$ & $\begin{array}{l}\text { slope }=0.011 \pm 0.005 \\
P=0.02 \\
\text { intercept } \\
\quad=0.06 \pm 0.04 \\
P=0.14 \\
R^{2}{ }_{t o t}=0.003 \\
n_{t o t}=1569\end{array}$ & $\begin{array}{l}P=0.97 \\
n_{\text {tot }}=545\end{array}$ & $\begin{array}{l}\text { slope }=0.05 \pm 0.02 \\
P=0.03 \\
\text { intercept } \\
\quad=-0.6 \pm 0.2 \\
P=0.03 \\
R^{2}{ }_{t o t}=0.124 \\
n_{\text {tot }}=28\end{array}$ \\
\hline & $\mathrm{S}$ & $\begin{array}{l}\text { slope }=0.10 \pm 0.05 \\
P=0.03 \\
\text { intercept } \\
\quad=-0.8 \pm 0.3 \\
P=0.02 \\
R_{\text {tot }}^{2}=0.072 \\
n_{\text {tot }}=123\end{array}$ & $\begin{array}{l}\text { slope }=0.011 \pm 0.005 \\
P=0.02 \\
\text { intercept } \\
\quad=0.06 \pm 0.04 \\
P=0.14 \\
R^{2} \text { tot }=0.003 \\
n_{\text {tot }}=1569\end{array}$ & $\begin{array}{l}P=0.97 \\
n_{\text {tot }}=545\end{array}$ & $\begin{array}{l}\text { slope }=0.05 \pm 0.02 \\
P=0.03 \\
\text { intercept } \\
\quad=-0.6 \pm 0.2 \\
P=0.03 \\
R^{2}{ }_{t o t}=0.124 \\
n_{\text {tot }}=28\end{array}$ \\
\hline $\begin{array}{l}\text { Actual/attainable } \\
\text { MAI } \\
(\text { method } 2)\end{array}$ & $\begin{array}{l}\text { entire } \\
\text { Sweden }\end{array}$ & $\begin{array}{l}P=0.80 \\
n=19\end{array}$ & $\begin{array}{l}\text { slope }=0.21 \pm 0.07 \\
P<0.01 \\
\text { intercept } \\
\quad=41.8 \pm 0.7 \\
P<0.01 \\
R^{2}=0.011 \\
n=641\end{array}$ & $\begin{array}{l}P=0.30 \\
n=285\end{array}$ & $\begin{array}{l}P=0.84 \\
n=10\end{array}$ \\
\hline
\end{tabular}


Table S5 (continued).

\begin{tabular}{|c|c|c|c|c|c|}
\hline \multirow[t]{2}{*}{$\begin{array}{l}\text { Normalized } \\
\text { productivity } \\
\text { response }\end{array}$} & \multirow[t]{2}{*}{ Region } & \multicolumn{4}{|l|}{$\begin{array}{l}\text { In TEB stock } \\
0-20 \mathrm{~cm} \\
{\left[\mathrm{cmol}_{+} \mathrm{m}^{-2}\right]}\end{array}$} \\
\hline & & Dry & Fresh & Fresh-moist & Moist \\
\hline \multirow[t]{3}{*}{$\begin{array}{l}\text { Residual MAI } \\
\text { (method 1) }\end{array}$} & $\mathrm{N}$ & $\begin{array}{l}\text { slope }=0.4 \pm 0.1 \\
P<0.01 \\
\text { intercept }=-1.7 \pm 0.3 \\
P<0.01 \\
R^{2}=0.274 \\
n=32\end{array}$ & $\begin{array}{l}\text { slope }=0.22 \pm 0.05 \\
P<0.01 \\
\text { intercept } \\
\quad=-0.6 \pm 0.2 \\
P<0.01 \\
R^{2}=0.039 \\
n=385\end{array}$ & $\begin{array}{l}\text { slope }=0.31 \pm 0.08 \\
P<0.01 \\
\text { intercept } \\
\quad=-1.5 \pm 0.3 \\
P<0.01 \\
R^{2}=0.111 \\
n=121\end{array}$ & $\begin{array}{l}\text { slope } \\
\quad=0.5 \pm 0.1 \\
P=0.02 \\
\text { intercept } \\
=-3.0 \pm 0.5 \\
P=0.01 \\
R^{2}=0.808 \\
n=4\end{array}$ \\
\hline & M & $\begin{array}{l}P=0.23 \\
n=47\end{array}$ & $\begin{array}{l}\text { slope }=0.20 \pm 0.07 \\
P<0.01 \\
\text { intercept } \\
\quad=-0.4 \pm 0.2 \\
P=0.09 \\
R^{2}=0.015 \\
n=515\end{array}$ & $\begin{array}{l}\text { slope }=0.18 \pm 0.09 \\
P=0.04 \\
\text { intercept } \\
\quad=-1.3 \pm 0.4 \\
P<0.01 \\
R^{2}=0.016 \\
n=208\end{array}$ & $\begin{array}{l}P=0.97 \\
n=7\end{array}$ \\
\hline & $\mathrm{S}$ & $\begin{array}{l}P=0.41 \\
n=44\end{array}$ & $\begin{array}{l}\text { slope }=-0.20 \pm 0.06 \\
P<0.01 \\
\text { intercept }=1.3 \pm 0.3 \\
P<0.01 \\
R^{2}=0.014 \\
n=669\end{array}$ & $\begin{array}{l}\text { slope }=0.4 \pm 0.1 \\
P<0.01 \\
\text { intercept } \\
\quad=-2.4 \pm 0.5 \\
P<0.01 \\
R^{2}=0.050 \\
n=216\end{array}$ & $\begin{array}{l}P=0.16 \\
n=17\end{array}$ \\
\hline $\begin{array}{l}\text { Actual/attainable } \\
\text { MAI } \\
(\operatorname{method} 2)\end{array}$ & $\begin{array}{l}\text { entire } \\
\text { Sweden }\end{array}$ & $\begin{array}{l}P=0.51 \\
n=19\end{array}$ & $\begin{array}{l}\text { slope }=2.7 \pm 0.7 \\
P<0.01 \\
\text { intercept } \\
\quad=32 \pm 3 \\
P<0.01 \\
R^{2}=0.025 \\
n=641\end{array}$ & $\begin{array}{l}\text { slope }=3.6 \pm 0.8 \\
P<0.01 \\
\text { intercept } \\
\quad=20 \pm 4 \\
P<0.01 \\
R^{2}=0.060 \\
n=285\end{array}$ & $\begin{array}{l}P=0.68 \\
n=10\end{array}$ \\
\hline
\end{tabular}


Table S5 (continued).

\begin{tabular}{|c|c|c|c|c|c|}
\hline \multirow{2}{*}{$\begin{array}{l}\text { Normalized } \\
\text { productivity } \\
\text { response }\end{array}$} & \multirow[t]{2}{*}{ Region } & \multicolumn{4}{|l|}{$\begin{array}{l}\text { pHкCl } \\
0-20 \mathrm{~cm}\end{array}$} \\
\hline & & Dry & Fresh & Fresh-moist & Moist \\
\hline \multirow[t]{3}{*}{$\begin{array}{l}\text { Residual MAI } \\
(\operatorname{method} 1)\end{array}$} & $\mathrm{N}$ & $\begin{array}{l}P=0.80 \\
n_{\text {tot }}=123\end{array}$ & $\begin{array}{l}\text { quad }=-0.54 \pm 0.07 \\
P<0.01 \\
\text { lin }=3.9 \pm 0.5 \\
P<0.01 \\
\text { intercept }=-7 \pm 1 \\
P<0.01 \\
R^{2} \text { tot }=0.043 \\
n_{t o t}=1569\end{array}$ & $\begin{array}{l}\text { quad }=-0.4 \pm 0.1 \\
P<0.01 \\
\text { lin }=3.4 \pm 0.8 \\
P<0.01 \\
\text { intercept }=-7 \pm 2 \\
P<0.01 \\
R^{2} \text { tot }=0.121 \\
n_{\text {tot }}=545\end{array}$ & $\begin{array}{l}\text { quad } \\
\quad=-1.8 \pm 0.8 \\
P=0.03 \\
\text { lin }=12 \pm 5 \\
P=0.03 \\
\text { intercept } \\
\quad=-22 \pm 9 \\
P=0.02 \\
R_{\text {tot }}^{2}=0.415 \\
n_{\text {tot }}=28\end{array}$ \\
\hline & M & $\begin{array}{l}P=0.80 \\
n_{\text {tot }}=123\end{array}$ & $\begin{array}{l}\text { quad }=-0.54 \pm 0.07 \\
P<0.01 \\
\text { lin }=4.2 \pm 0.5 \\
P<0.01 \\
\text { intercept }=-8 \pm 1 \\
P<0.01 \\
R^{2}{ }_{t o t}=0.043 \\
n_{t o t}=1569\end{array}$ & $\begin{array}{l}\text { quad }=-0.4 \pm 0.1 \\
P<0.01 \\
\text { lin }=3.5 \pm 0.8 \\
P<0.01 \\
\text { intercept }=-8 \pm 1 \\
P<0.01 \\
R^{2} \text { tot }=0.121 \\
n_{\text {tot }}=545\end{array}$ & $\begin{array}{l}\text { quad } \\
\quad=-1.8 \pm 0.8 \\
P=0.03 \\
\text { lin }=12 \pm 5 \\
P=0.03 \\
\text { intercept } \\
\quad=-23 \pm 9 \\
P=0.02 \\
R_{\text {tot }}^{2}=0.415 \\
n_{\text {tot }}=28\end{array}$ \\
\hline & $\mathrm{S}$ & $\begin{array}{l}P=0.80 \\
n_{t o t}=123\end{array}$ & $\begin{array}{l}\text { quad }=-0.54 \pm 0.07 \\
P<0.01 \\
\text { lin }=4.2 \pm 0.5 \\
P<0.01 \\
\text { intercept }=-8 \pm 1 \\
P<0.01 \\
R^{2}{ }_{t o t}=0.043 \\
n_{\text {tot }}=1569\end{array}$ & $\begin{array}{l}\text { quad }=-0.4 \pm 0.1 \\
P<0.01 \\
\text { lin }=3.8 \pm 0.8 \\
P<0.01 \\
\text { intercept }=-8 \pm 1 \\
P<0.01 \\
R^{2} \text { tot }=0.121 \\
n_{t o t}=545\end{array}$ & $\begin{array}{l}\text { quad } \\
\quad=-1.8 \pm 0.8 \\
P=0.03 \\
\text { lin }=12 \pm 5 \\
P=0.03 \\
\text { intercept } \\
\quad=-21 \pm 9 \\
P=0.03 \\
R_{\text {tot }}^{2}=0.415 \\
n_{\text {tot }}=28\end{array}$ \\
\hline $\begin{array}{l}\text { Actual/attainable } \\
\text { MAI } \\
\text { (method 2) }\end{array}$ & $\begin{array}{l}\text { entire } \\
\text { Sweden }\end{array}$ & $\begin{array}{l}P=0.13 \\
n=19\end{array}$ & $\begin{array}{l}P=0.21 \\
n=641\end{array}$ & $\begin{array}{l}\text { slope }=4 \pm 2 \\
P=0.02 \\
\text { intercept } \\
\quad=23 \pm 5 \\
P<0.01 \\
R^{2}=0.016 \\
n=285\end{array}$ & $\begin{array}{l}P=0.45 \\
n=10\end{array}$ \\
\hline
\end{tabular}


Table S6. Associations between single soil variables and normalized productivity of Table 2, stratified by soil type. Significance $(P$ values) of single soil variable effects on residual productivity (mean annual increment - MAI [m $\left.\mathrm{ma}^{3} \mathrm{yr}^{-1}\right]$ ) and actual/attainable MAI (for spruce only) across Sweden are given. For (near) significant variables (i.e. $P<0.10$ ), parameter estimates \pm s.e.m. and the proportion of variation explained $\left(R^{2}\right)$ are shown as well. Abbreviations: $\mathrm{N}=$ north; $\mathrm{M}=$ middle; $\mathrm{S}=$ south; $\mathrm{SOC}=$ soil organic carbon concentration; Soil C:N = soil carbon to nitrogen ratio; TEB = total exchangeable bases; quad = parameter estimate for quadratic term; lin $=$ parameter estimate for linear term of a quadratic function.

\begin{tabular}{|c|c|c|c|c|c|c|}
\hline \multirow[t]{2}{*}{$\begin{array}{l}\text { Normalized } \\
\text { productivity } \\
\text { response }\end{array}$} & \multirow[t]{2}{*}{ Region } & \multicolumn{5}{|l|}{$\begin{array}{l}\text { In SOC } \\
0-20 \mathrm{~cm} \\
{[\%]}\end{array}$} \\
\hline & & Histosol & Gleysol & Regosol & Leptosol & Podzol \\
\hline \multirow[t]{3}{*}{$\begin{array}{l}\text { Residual MAI } \\
\text { (method } 1 \text { ) }\end{array}$} & $\mathrm{N}$ & $\begin{array}{l}\text { quad }=-1.9 \pm 0.9 \\
P=0.04 \\
\text { lin }=11 \pm 7 \\
P=0.15 \\
\text { intercept } \\
\quad=-14 \pm 17 \\
P=0.42 \\
R^{2} \text { tot }=0.063 \\
n_{\text {tot }}=156\end{array}$ & $\begin{array}{l}P=0.66 \\
n=30\end{array}$ & $\begin{array}{l}\text { quad }=-0.12 \pm 0.03 \\
P<0.01 \\
\text { lin }=0.3 \pm 0.1 \\
P=0.02 \\
\text { intercept } \\
\quad=-0.1 \pm 0.1 \\
P=0.64 \\
R^{2}{ }_{t o t}=0.064 \\
n_{t o t}=823\end{array}$ & $\begin{array}{l}P=0.46 \\
n_{t o t}=71\end{array}$ & $\begin{array}{l}\text { quad }=-0.12 \pm 0.03 \\
P<0.01 \\
\text { lin }=0.4 \pm 0.1 \\
P<0.01 \\
\text { intercept } \\
\quad=-0.1 \pm 0.1 \\
P=0.26 \\
R^{2}{ }_{\text {tot }}=0.043 \\
n_{\text {tot }}=979\end{array}$ \\
\hline & M & $\begin{array}{l}\text { quad }=-1.9 \pm 0.9 \\
P=0.04 \\
\text { lin }=12 \pm 7 \\
P=0.07 \\
\text { intercept } \\
\quad=-20 \pm 13 \\
P=0.11 \\
R^{2}{ }_{t o t}=0.063 \\
n_{t o t}=156\end{array}$ & $\begin{array}{l}P=0.56 \\
n=28\end{array}$ & $\begin{array}{l}\text { quad }=-0.12 \pm 0.03 \\
P<0.01 \\
\text { lin }=0.2 \pm 0.1 \\
P=0.07 \\
\text { intercept } \\
\quad=0.1 \pm 0.1 \\
P=0.39 \\
R_{\text {tot }}^{2}=0.064 \\
n_{\text {tot }}=823\end{array}$ & $\begin{array}{l}P=0.46 \\
n_{\text {tot }}=71\end{array}$ & $\begin{array}{l}\text { quad }=-0.12 \pm 0.03 \\
P<0.01 \\
\text { lin }=0.3 \pm 0.1 \\
P=0.03 \\
\text { intercept } \\
\quad=0.0 \pm 0.1 \\
P=0.68 \\
R_{\text {tot }}^{2}=0.043 \\
n_{\text {tot }}=979\end{array}$ \\
\hline & $\mathrm{S}$ & $\begin{array}{l}\text { quad }=-1.9 \pm 0.9 \\
P=0.04 \\
\text { lin }=10 \pm 5 \\
P=0.07 \\
\text { intercept } \\
\quad=-12 \pm 8 \\
P=0.14 \\
R_{\text {tot }}^{2}=0.063 \\
n_{\text {tot }}=156\end{array}$ & $\begin{array}{l}\text { slope }=-0.6 \pm \\
0.2 \\
P<0.01 \\
\text { intercept } \\
\quad=0.9 \pm 0.6 \\
P=0.11 \\
R^{2}=0.183 \\
n=34\end{array}$ & $\begin{array}{l}\text { quad }=-0.12 \pm 0.03 \\
P=0.27 \\
\text { lin }=0.2 \pm 0.1 \\
P=0.23 \\
\text { intercept } \\
\quad=0.5 \pm 0.2 \\
P<0.01 \\
R_{\text {tot }}^{2}=0.064 \\
n_{\text {tot }}=823\end{array}$ & $\begin{array}{l}P=0.46 \\
n_{t o t}=71\end{array}$ & $\begin{array}{l}\text { quad }=-0.12 \pm 0.03 \\
P<0.01 \\
\text { lin }=0.2 \pm 0.1 \\
P=0.19 \\
\text { intercept } \\
\quad=0.4 \pm 0.2 \\
P=0.01 \\
R_{\text {tot }}^{2}=0.043 \\
n_{\text {tot }}=979\end{array}$ \\
\hline $\begin{array}{l}\text { Actual/attainable } \\
\text { MAI } \\
\text { (method } 2)\end{array}$ & $\begin{array}{l}\text { entire } \\
\text { Sweden }\end{array}$ & $\begin{array}{l}\text { slope }=-11 \pm 5 \\
P=0.05 \\
\text { intercept } \\
\quad=75 \pm 20 \\
P<0.01 \\
R^{2}=0.051 \\
n=56\end{array}$ & $\begin{array}{l}P=0.55 \\
n=59\end{array}$ & $\begin{array}{l}\text { quad }=-1.9 \pm 0.7 \\
P<0.01 \\
\text { lin }=8 \pm 3 \\
P<0.01 \\
\text { intercept } \\
\quad=35 \pm 3 \\
P<0.01 \\
R^{2}=0.016 \\
n=350\end{array}$ & $\begin{array}{l}\text { quad }=-3 \pm 1 \\
P=0.01 \\
\text { lin }=16 \pm 5 \\
P<0.01 \\
\text { intercept } \\
\quad=20 \pm 6 \\
P<0.01 \\
R^{2}=0.199 \\
n=27\end{array}$ & $\begin{array}{l}\text { quad }=-2.3 \pm 0.6 \\
P<0.01 \\
\text { lin }=10 \pm 2 \\
P<0.01 \\
\text { intercept } \\
\quad=31 \pm 2 \\
P<0.01 \\
R^{2}=0.041 \\
n=390\end{array}$ \\
\hline
\end{tabular}


Table S6 (continued).

\begin{tabular}{|c|c|c|c|c|c|c|}
\hline \multirow[t]{2}{*}{$\begin{array}{l}\text { Normalized } \\
\text { productivity } \\
\text { response }\end{array}$} & \multirow[t]{2}{*}{ Region } & \multicolumn{5}{|l|}{$\begin{array}{l}\text { In N stock } \\
0-20 \mathrm{~cm} \\
{\left[\mathrm{~g} \mathrm{~m}^{-2}\right]}\end{array}$} \\
\hline & & Histosol & Gleysol & Regosol & Leptosol & Podzol \\
\hline \multirow[t]{3}{*}{$\begin{array}{l}\text { Residual MAI } \\
(\operatorname{method} 1)\end{array}$} & $\mathrm{N}$ & $\begin{array}{l}\text { slope }=0.9 \pm 0.2 \\
P<0.01 \\
\text { intercept } \\
\quad=-6.3 \pm 0.9 \\
P<0.01 \\
R^{2} \text { tot }=0.140 \\
n_{\text {tot }}=158\end{array}$ & $\begin{array}{l}P=0.62 \\
n_{\text {tot }}=91\end{array}$ & $\begin{array}{l}\text { slope }=0.23 \pm 0.08 \\
P<0.01 \\
\text { intercept } \\
\quad=-1.0 \pm 0.4 \\
P=0.02 \\
R^{2}{ }_{t o t}=0.008 \\
n_{t o t}=825\end{array}$ & $\begin{array}{l}P=0.95 \\
n_{\text {tot }}=73\end{array}$ & $\begin{array}{l}\text { slope }=0.28 \pm 0.08 \\
P<0.01 \\
\text { intercept }=-1.2 \pm 0.4 \\
P<0.01 \\
R^{2}{ }_{t o t}=0.012 \\
n_{t o t}=981\end{array}$ \\
\hline & $\mathrm{M}$ & $\begin{array}{l}\text { slope }=0.9 \pm 0.2 \\
P<0.01 \\
\text { intercept } \\
\quad=-6.3 \pm 0.9 \\
P<0.01 \\
R^{2} \text { tot }=0.140 \\
n_{t o t}=158\end{array}$ & $\begin{array}{l}P=0.62 \\
n_{\text {tot }}=91\end{array}$ & $\begin{array}{l}\text { slope }=0.23 \pm 0.08 \\
P<0.01 \\
\text { intercept } \\
\quad=-1.0 \pm 0.4 \\
P=0.02 \\
R^{2}{ }_{t o t}=0.008 \\
n_{\text {tot }}=825\end{array}$ & $\begin{array}{l}P=0.95 \\
n_{t o t}=73\end{array}$ & $\begin{array}{l}\text { slope }=0.28 \pm 0.08 \\
P<0.01 \\
\text { intercept }=-1.2 \pm 0.4 \\
P<0.01 \\
R^{2} \text { tot }=0.012 \\
n_{t o t}=981\end{array}$ \\
\hline & $\mathrm{S}$ & $\begin{array}{l}\text { slope }=0.9 \pm 0.2 \\
P<0.01 \\
\text { intercept } \\
\quad=-6.3 \pm 0.9 \\
P<0.01 \\
R^{2} \text { tot }=0.140 \\
n_{\text {tot }}=158\end{array}$ & $\begin{array}{l}P=0.62 \\
n_{t o t}=91\end{array}$ & $\begin{array}{l}\text { slope }=0.23 \pm 0.08 \\
P<0.01 \\
\text { intercept } \\
\quad=-1.0 \pm 0.4 \\
P=0.02 \\
R^{2} \text { tot }=0.008 \\
n_{\text {tot }}=825\end{array}$ & $\begin{array}{l}P=0.95 \\
n_{\text {tot }}=73\end{array}$ & $\begin{array}{l}\text { slope }=0.28 \pm 0.08 \\
P<0.01 \\
\text { intercept }=-1.2 \pm 0.4 \\
P<0.01 \\
R^{2} \text { tot }=0.012 \\
n_{t o t}=981\end{array}$ \\
\hline $\begin{array}{l}\text { Actual/attainable } \\
\text { MAI } \\
(\text { method } 2)\end{array}$ & $\begin{array}{l}\text { entire } \\
\text { Sweden }\end{array}$ & $\begin{array}{l}\text { slope }=10 \pm 3 \\
P<0.01 \\
\text { intercept } \\
\quad=-26 \pm 15 \\
P=0.10 \\
R^{2}=0.213 \\
n=56\end{array}$ & $\begin{array}{l}\text { slope }=8 \pm 3 \\
P<0.01 \\
\text { intercept } \\
\quad=-9 \pm 14 \\
P=0.54 \\
R^{2}=0.131 \\
n=59\end{array}$ & $\begin{array}{l}\text { slope }=12 \pm 1 \\
P<0.01 \\
\text { intercept } \\
\quad=-25 \pm 8 \\
P<0.01 \\
R^{2}=0.178 \\
n=350\end{array}$ & $\begin{array}{l}\text { slope }=18 \pm 3 \\
P<0.01 \\
\text { intercept } \\
\quad=-64 \pm 21 \\
P<0.01 \\
R^{2}=0.442 \\
n=27\end{array}$ & $\begin{array}{l}\text { slope }=12 \pm 2 \\
P<0.01 \\
\text { intercept } \\
\quad=-24 \pm 9 \\
P<0.01 \\
R^{2}=0.115 \\
n=390\end{array}$ \\
\hline
\end{tabular}


Table S6 (continued).

\begin{tabular}{|c|c|c|c|c|c|c|}
\hline \multirow{2}{*}{$\begin{array}{l}\text { Normalized } \\
\text { productivity } \\
\text { response }\end{array}$} & \multirow[t]{2}{*}{ Region } & \multicolumn{5}{|l|}{$\begin{array}{l}\text { Soil C:N } \\
0-20 \mathrm{~cm}\end{array}$} \\
\hline & & Histosol & Gleysol & Regosol & Leptosol & Podzol \\
\hline \multirow[t]{3}{*}{$\begin{array}{l}\text { Residual MAI } \\
\text { (method 1) }\end{array}$} & $\mathrm{N}$ & $\begin{array}{l}\text { slope } \\
\quad=-0.06 \pm 0.02 \\
P=0.04 \\
\text { intercept } \\
\quad=0.6 \pm 0.7 \\
P=0.40 \\
R^{2}=0.255 \\
n=13\end{array}$ & $\begin{array}{l}\text { slope } \\
\quad=-0.03 \pm 0.01 \\
P=0.03 \\
\text { intercept } \\
\quad=0.6 \pm 0.3 \\
P=0.10 \\
R^{2}=0.121 \\
n=30\end{array}$ & $\begin{array}{l}P=0.13 \\
n=203\end{array}$ & $\begin{array}{l}P=0.45 \\
n_{\text {tot }}=71\end{array}$ & $\begin{array}{l}\text { slope } \\
\quad=-0.013 \pm 0.006 \\
P=0.03 \\
\text { intercept }=0.4 \pm 0.2 \\
P=0.01 \\
R^{2}=0.015 \\
n=267\end{array}$ \\
\hline & M & $\begin{array}{l}\text { slope } \\
\quad=-0.05 \pm 0.02 \\
P=0.03 \\
\text { intercept } \\
\quad=0.0 \pm 0.6 \\
P=0.99 \\
R^{2}=0.079 \\
n=50\end{array}$ & $\begin{array}{l}P=0.11 \\
n=25\end{array}$ & $\begin{array}{l}\text { slope } \\
\quad=-0.016 \pm 0.009 \\
P=0.07 \\
\text { intercept } \\
\quad=0.5 \pm 0.2 \\
P=0.04 \\
R^{2}=0.008 \\
n=262\end{array}$ & $\begin{array}{l}P=0.45 \\
n_{t o t}=71\end{array}$ & $\begin{array}{l}\text { slope } \\
\quad=-0.026 \pm 0.008 \\
P<0.01 \\
\text { intercept }=0.8 \pm 0.2 \\
P<0.01 \\
R^{2}=0.027 \\
n=390\end{array}$ \\
\hline & $\mathrm{S}$ & $\begin{array}{l}\text { slope } \\
\quad=-0.10 \pm 0.02 \\
P<0.01 \\
\text { intercept } \\
\quad=1.3 \pm 0.7 \\
P=0.06 \\
R^{2}=0.154 \\
n=93\end{array}$ & $\begin{array}{l}P=0.40 \\
n=34\end{array}$ & $\begin{array}{l}\text { slope }=-0.07 \pm 0.01 \\
P<0.01 \\
\text { intercept } \\
\quad=1.8 \pm 0.3 \\
P<0.01 \\
R^{2}=0.067 \\
n=358\end{array}$ & $\begin{array}{l}P=0.45 \\
n_{t o t}=71\end{array}$ & $\begin{array}{l}\text { slope }=-0.04 \pm 0.01 \\
P<0.01 \\
\text { intercept }=1.3 \pm 0.3 \\
P<0.01 \\
R^{2}=0.027 \\
n=322\end{array}$ \\
\hline $\begin{array}{l}\text { Actual/attainable } \\
\text { MAI } \\
(\operatorname{method} 2)\end{array}$ & $\begin{array}{l}\text { entire } \\
\text { Sweden }\end{array}$ & N/A & N/A & N/A & N/A & N/A \\
\hline
\end{tabular}


Table S6 (continued).

\begin{tabular}{|c|c|c|c|c|c|c|}
\hline \multirow{2}{*}{$\begin{array}{l}\text { Normalized } \\
\text { productivity } \\
\text { response }\end{array}$} & \multirow[t]{2}{*}{ Region } & \multicolumn{5}{|l|}{$\begin{array}{l}\text { In soil C:N } \\
0-10 \mathrm{~cm}\end{array}$} \\
\hline & & Histosol & Gleysol & Regosol & Leptosol & Podzol \\
\hline \multirow{3}{*}{$\begin{array}{l}\text { Residual MAI } \\
(\operatorname{method} 1)\end{array}$} & $\mathrm{N}$ & N/A & N/A & N/A & N/A & N/A \\
\hline & M & N/A & N/A & N/A & N/A & N/A \\
\hline & $\mathrm{S}$ & N/A & N/A & N/A & N/A & N/A \\
\hline \multirow{7}{*}{$\begin{array}{l}\text { Actual/attainable } \\
\text { MAI } \\
(\text { method } 2)\end{array}$} & entire & slope $=-25 \pm 8$ & slope $=-17 \pm 4$ & slope $=-20 \pm 2$ & slope $=-19 \pm 9$ & slope $=-16 \pm 3$ \\
\hline & Sweden & $P<0.01$ & $P<0.01$ & $P<0.01$ & $P=0.03$ & $P<0.01$ \\
\hline & & intercept & intercept & intercept & intercept & intercept \\
\hline & & $=113 \pm 26$ & $=90 \pm 14$ & $=106 \pm 8$ & $=102 \pm 29$ & $=91 \pm 9$ \\
\hline & & $P<0.01$ & $P<0.01$ & $P<0.01$ & $P<0.01$ & $P<0.01$ \\
\hline & & $R^{2}=0.117$ & $R^{2}=0.176$ & $R^{2}=0.154$ & $R^{2}=0.093$ & $R^{2}=0.082$ \\
\hline & & $n=61$ & $n=62$ & $n=360$ & $n=38$ & $n=391$ \\
\hline
\end{tabular}


Table S6 (continued).

\begin{tabular}{lll}
\hline $\begin{array}{l}\text { Normalized } \\
\text { productivity } \\
\text { response }\end{array}$ & Region & $\begin{array}{l}\text { Mineral soil sand } \\
{[\%]}\end{array}$ \\
& &
\end{tabular}

\section{Residual MAI}

(method 1)

\section{Histosol}

N

$P=0.44$

$n_{\text {tot }}=174$

M

$$
\begin{aligned}
& P=0.44 \\
& n_{\text {tot }}=174
\end{aligned}
$$

S

$$
\begin{aligned}
& P=0.44 \\
& n_{\text {tot }}=174
\end{aligned}
$$

entire

Sweden

$P=0.75$

\section{MAI}

(method 2)
$P=0.21$
$n_{\text {tot }}=95$

Gleysol
$P=0.21$

$n_{\text {tot }}=95$

$P=0.74$

$n=217$

$P=0.98$
$n=277$

$P=0.21$

$n_{\text {tot }}=95$

$P=0.65$

$n=63$

$P=0.93$

$n=374$

$P=0.05$
Leptosol

$P=0.48$

$n_{\text {tot }}=181$

$P=0.48$

$n_{\text {tot }}=181$

slope $=-0.06 \pm 0.03$

intercept

$=45 \pm 2$

$P<0.01$

$R^{2}=0.008$

$n=377$
Podzol

slope

$=-0.004 \pm 0.002$

$P=0.09$

intercept

$=0.3 \pm 0.2$

$P=0.04$

$R^{2}=0.007$

$n=268$

slope $=0.008 \pm 0.003$

$P<0.01$

intercept

$=-0.4 \pm 0.2$

$P=0.05$

$R^{2}=0.016$

$n=401$

$P=0.69$

$n=324$

slope $=-0.06 \pm 0.03$

$P=0.03$

intercept

$=43 \pm 2$

$P<0.01$

$R^{2}=0.009$

$n=399$ 
Table S6 (continued).

\begin{tabular}{lll}
\hline $\begin{array}{l}\text { Normalized } \\
\text { productivity } \\
\text { response }\end{array}$ & Region & Mineral soil clay \\
{$[\%]$}
\end{tabular}

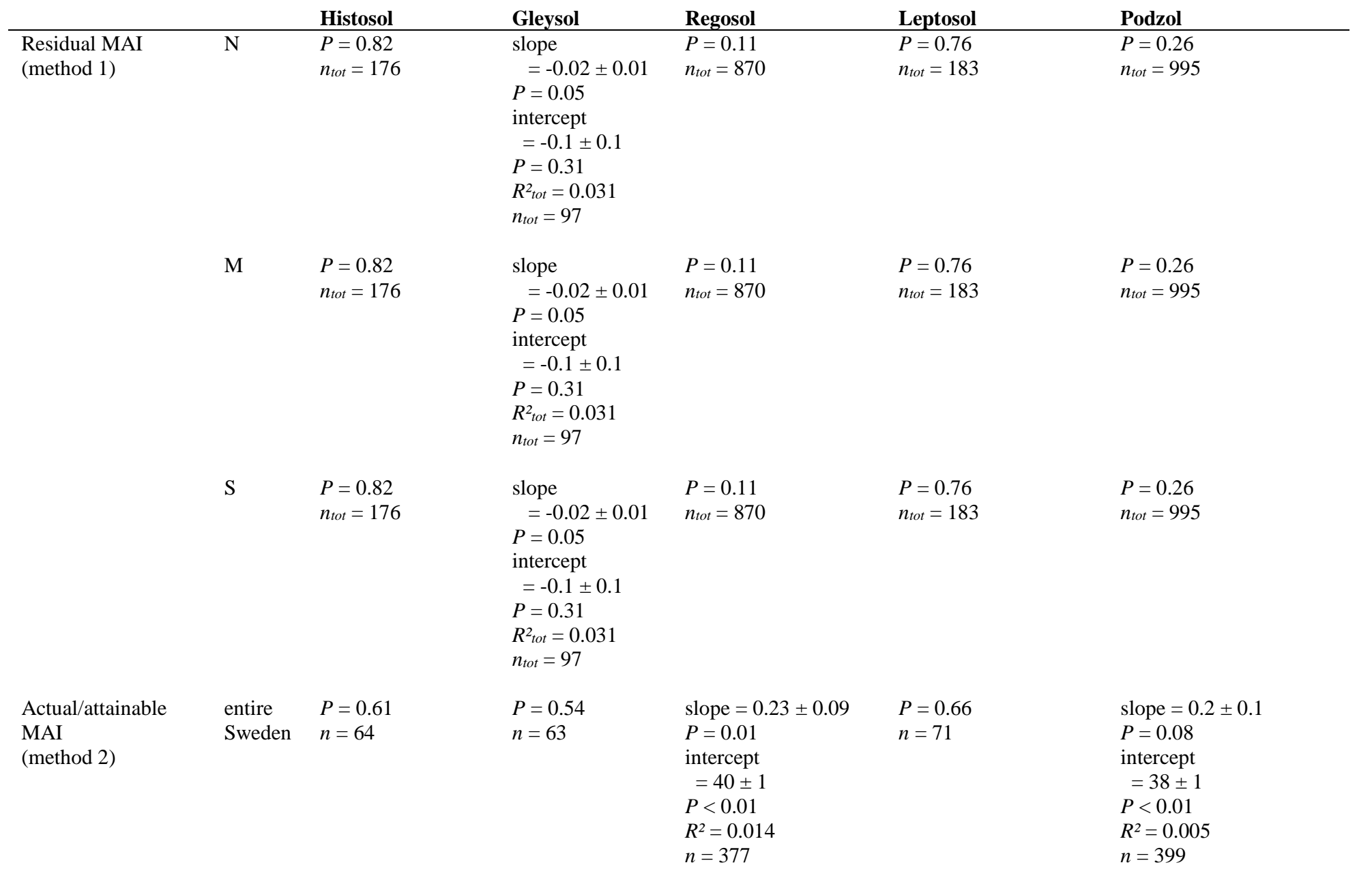


Table S6 (continued).

\begin{tabular}{|c|c|c|c|c|c|c|}
\hline \multirow[t]{2}{*}{$\begin{array}{l}\text { Normalized } \\
\text { productivity } \\
\text { response }\end{array}$} & \multirow[t]{2}{*}{ Region } & \multicolumn{5}{|l|}{$\begin{array}{l}\text { In TEB stock } \\
0-20 \mathrm{~cm} \\
{\left[\mathrm{cmol}_{+} \mathrm{m}^{-2}\right]}\end{array}$} \\
\hline & & Histosol & Gleysol & Regosol & Leptosol & Podzol \\
\hline \multirow[t]{3}{*}{$\begin{array}{l}\text { Residual MAI } \\
\text { (method } 1 \text { ) }\end{array}$} & $\mathrm{N}$ & $\begin{array}{l}P=0.27 \\
n=56\end{array}$ & $\begin{array}{l}P=0.17 \\
n_{\text {tot }}=89\end{array}$ & $\begin{array}{l}\text { slope }=0.25 \pm 0.06 \\
P<0.01 \\
\text { intercept } \\
\quad=-0.9 \pm 0.2 \\
P<0.01 \\
R^{2}=0.070 \\
n=203\end{array}$ & $\begin{array}{l}P=0.21 \\
n_{\text {tot }}=71\end{array}$ & $\begin{array}{l}\text { slope }=0.23 \pm 0.08 \\
P<0.01 \\
\text { intercept } \\
\quad=-0.7 \pm 0.3 \\
P<0.01 \\
R^{2}=0.029 \\
n=266\end{array}$ \\
\hline & M & $\begin{array}{l}P=0.51 \\
n=50\end{array}$ & $\begin{array}{l}P=0.17 \\
n_{\text {tot }}=89\end{array}$ & $\begin{array}{l}\text { slope }=0.25 \pm 0.09 \\
P<0.01 \\
\text { intercept } \\
\quad=-0.9 \pm 0.3 \\
P=0.01 \\
R^{2}=0.026 \\
n=262\end{array}$ & $\begin{array}{l}P=0.21 \\
n_{t o t}=71\end{array}$ & $\begin{array}{l}\text { slope }=0.15 \pm 0.08 \\
P=0.08 \\
\text { intercept } \\
=-0.4 \pm 0.3 \\
P=0.18 \\
R^{2}=0.005 \\
n=390\end{array}$ \\
\hline & $S$ & $\begin{array}{l}\text { slope }=0.7 \pm 0.2 \\
P<0.01 \\
\text { intercept } \\
\quad=-4.4 \pm 0.9 \\
P<0.01 \\
R^{2}=0.096 \\
n=93\end{array}$ & $\begin{array}{l}P=0.17 \\
n_{\text {tot }}=89\end{array}$ & $\begin{array}{l}\text { slope }=-0.15 \pm 0.09 \\
P=0.08 \\
\text { intercept } \\
\quad=1.0 \pm 0.4 \\
P<0.01 \\
R^{2}=0.006 \\
n=358\end{array}$ & $\begin{array}{l}P=0.21 \\
n_{\text {tot }}=71\end{array}$ & $\begin{array}{l}P=0.6 \\
n=322\end{array}$ \\
\hline $\begin{array}{l}\text { Actual/attainable } \\
\text { MAI } \\
(\text { method } 2)\end{array}$ & $\begin{array}{l}\text { entire } \\
\text { Sweden }\end{array}$ & $\begin{array}{l}P=0.13 \\
n=56\end{array}$ & $\begin{array}{l}P=0.30 \\
n=59\end{array}$ & $\begin{array}{l}\text { slope }=1.4 \pm 0.8 \\
P=0.09 \\
\text { intercept } \\
\quad=36 \pm 4 \\
P<0.01 \\
R^{2}=0.005 \\
n=350\end{array}$ & $\begin{array}{l}P=0.15 \\
n=27\end{array}$ & $\begin{array}{l}\text { slope }=2 \pm 1 \\
P=0.09 \\
\text { intercept } \\
\quad=32 \pm 4 \\
P<0.01 \\
R^{2}=0.005 \\
n=390\end{array}$ \\
\hline
\end{tabular}


Table S6 (continued).

\begin{tabular}{|c|c|c|c|c|c|c|}
\hline \multirow{2}{*}{$\begin{array}{l}\text { Normalized } \\
\text { productivity } \\
\text { response }\end{array}$} & \multirow[t]{2}{*}{ Region } & \multicolumn{5}{|l|}{$\begin{array}{l}\mathrm{pH} \\
0-20 \mathrm{~cm}\end{array}$} \\
\hline & & Histosol & Gleysol & Regosol & Leptosol & Podzol \\
\hline \multirow[t]{3}{*}{$\begin{array}{l}\text { Residual MAI } \\
(\operatorname{method} 1)\end{array}$} & $\mathrm{N}$ & $\begin{array}{l}\text { quad }=-0.1 \pm 0.3 \\
P=0.65 \\
\text { lin }=2 \pm 2 \\
P=0.48 \\
\text { intercept } \\
=0 \pm 2 \\
P=0.84 \\
R^{2}{ }_{\text {tot }}=0.037 \\
n_{\text {tot }}=156\end{array}$ & $\begin{array}{l}\text { quad }=-0.3 \pm 0.2 \\
P=0.23 \\
\text { lin }=2 \pm 2 \\
P=0.19 \\
\text { intercept } \\
\quad=-5 \pm 4 \\
P=0.16 \\
R_{\text {tot }}^{2}=0.099 \\
n_{\text {tot }}=89\end{array}$ & $\begin{array}{l}\text { quad }=-0.45 \pm 0.09 \\
P<0.01 \\
\text { lin }=3.4 \pm 0.7 \\
P<0.01 \\
\text { intercept } \\
\quad=-6 \pm 1 \\
P<0.01 \\
R_{\text {tot }}=0.049 \\
n_{\text {tot }}=822\end{array}$ & $\begin{array}{l}P=0.42 \\
n_{\text {tot }}=71\end{array}$ & $\begin{array}{l}\text { quad }=-0.6 \pm 0.2 \\
P<0.01 \\
\text { lin }=4 \pm 1 \\
P<0.01 \\
\text { intercept } \\
=-7 \pm 2 \\
P<0.01 \\
R_{\text {tot }}^{2}=0.029 \\
n_{\text {tot }}=977\end{array}$ \\
\hline & $\mathrm{M}$ & $\begin{array}{l}\text { quad }=-0.1 \pm 0.3 \\
P=0.65 \\
\text { lin }=1 \pm 2 \\
P=0.55 \\
\text { intercept } \\
\quad=-4 \pm 4 \\
P=0.28 \\
R^{2} \text { tot }=0.037 \\
n_{\text {tot }}=156\end{array}$ & $\begin{array}{l}\text { quad }=-0.3 \pm 0.2 \\
P=0.23 \\
\text { lin }=4 \pm 2 \\
P=0.05 \\
\text { intercept } \\
\quad=-10 \pm 4 \\
P=0.01 \\
R^{2} \text { tot }=0.099 \\
n_{\text {tot }}=89\end{array}$ & $\begin{array}{l}\text { quad }=-0.45 \pm 0.09 \\
P<0.01 \\
\text { lin }=3.9 \pm 0.7 \\
P<0.01 \\
\text { intercept } \\
\quad=-8 \pm 1 \\
P<0.01 \\
R_{\text {tot }}^{2}=0.049 \\
n_{\text {tot }}=822\end{array}$ & $\begin{array}{l}P=0.42 \\
n_{t o t}=71\end{array}$ & $\begin{array}{l}\text { quad }=-0.6 \pm 0.2 \\
P<0.01 \\
\text { lin }=4 \pm 1 \\
P<0.01 \\
\text { intercept } \\
\quad=-7 \pm 2 \\
P<0.01 \\
R^{2} \text { tot }=0.029 \\
n_{\text {tot }}=977\end{array}$ \\
\hline & $\mathrm{S}$ & $\begin{array}{l}\text { quad }=-0.1 \pm 0.3 \\
P=0.65 \\
\text { lin }=2 \pm 2 \\
P=0.36 \\
\text { intercept } \\
\quad=-2 \pm 1 \\
P=0.30 \\
R_{\text {tot }}^{2}=0.037 \\
n_{\text {tot }}=156\end{array}$ & $\begin{array}{l}\text { quad }=-0.3 \pm 0.2 \\
P=0.23 \\
\text { lin }=3 \pm 2 \\
P=0.16 \\
\text { intercept } \\
\quad=-7 \pm 4 \\
P=0.08 \\
R_{\text {tot }}^{2}=0.099 \\
n_{\text {tot }}=89\end{array}$ & $\begin{array}{l}\text { quad }=-0.45 \pm 0.09 \\
P<0.01 \\
\text { lin }=3.6 \pm 0.7 \\
P<0.01 \\
\text { intercept } \\
\quad=-8 \pm 1 \\
P<0.01 \\
R_{\text {tot }}=0.049 \\
n_{\text {tot }}=822\end{array}$ & $\begin{array}{l}P=0.42 \\
n_{\text {tot }}=71\end{array}$ & $\begin{array}{l}\text { quad }=-0.6 \pm 0.2 \\
P<0.01 \\
\text { lin }=5 \pm 1 \\
P<0.01 \\
\text { intercept } \\
\quad=-8 \pm 2 \\
P<0.01 \\
R^{2} \text { tot }=0.029 \\
n_{\text {tot }}=977\end{array}$ \\
\hline $\begin{array}{l}\text { Actual/attainable } \\
\text { MAI }\end{array}$ & $\begin{array}{l}\text { entire } \\
\text { Sweden }\end{array}$ & $\begin{array}{l}P=0.42 \\
n=56\end{array}$ & $\begin{array}{l}P=0.50 \\
n=59\end{array}$ & $\begin{array}{l}P=0.11 \\
n=350\end{array}$ & $\begin{array}{l}P=0.63 \\
n=27\end{array}$ & $\begin{array}{l}P=0.92 \\
n=389\end{array}$ \\
\hline
\end{tabular}


Table S7. Overall mean squared error (ms) after 10-fold cross-validation for the most relevant candidate model structures that explain 140 variation in normalized productivity according to method 1 (residual mean annual increment - MAI) by soil variables $(0-20 \mathrm{~cm}$ depth or mineral soil only) for Swedish spruce and pine forests. The selected model is marked in gray. Abbreviations: C: $\mathrm{N}=$ soil carbon to nitrogen ratio; $\mathrm{pH}=\mathrm{pH}_{\mathrm{KCl}} ; \mathrm{SOC}=$ soil organic carbon concentration; $\mathrm{CLAY}_{\text {min.soil }}=$ clay fraction in the mineral soil; TEB = total exchangeable bases. REGION is a factor with north, middle and south as levels.

\begin{tabular}{|c|c|c|c|}
\hline Variables in model & $\begin{array}{c}\text { Overall } \\
\text { ms }\end{array}$ & Variables in model & $\begin{array}{c}\text { Overall } \\
\text { ms }\end{array}$ \\
\hline $\begin{array}{l}\mathrm{LN}^{2}(\mathrm{SOC}), \mathrm{LN}(\mathrm{SOC}), \mathrm{C}: \mathrm{N}, \mathrm{CLAY}_{\text {min.soil }}, \mathrm{LN}(\mathrm{TEB}), \\
\mathrm{pH}^{2}, \mathrm{pH}, \mathrm{REGION}, \\
\text { all interactions with REGION }\end{array}$ & 1.24 & \multirow{2}{*}{$\begin{array}{l}L N^{2}(S O C), L N(S O C), C: N, L N(T E B), p H^{2}, \\
p H, R E G I O N, \\
\text { all interactions with REGION except } \\
L N^{2}(\text { SOC):REGION, } \\
L N(S O C): R E G I O N, \\
L N(T E B): R E G I O N\end{array}$} & 1.23 \\
\hline $\begin{array}{l}\mathrm{LN}^{2} \text { (SOC), LN(SOC), C:N, CLAYmin.soil, LN(TEB), } \\
\mathrm{pH}^{2} \text {, pH, REGION, } \\
\text { all interactions with REGION except } \\
\text { LN }^{2}(\mathrm{SOC}): \text { REGION }\end{array}$ & 1.24 & & \\
\hline $\begin{array}{l}\mathrm{LN}^{2}(\mathrm{SOC}), \mathrm{LN}(\mathrm{SOC}), \mathrm{C}: \mathrm{N}, \mathrm{CLAY}_{\text {min.soil, }} \mathrm{LN}(\mathrm{TEB}) \text {, } \\
\mathrm{pH}^{2}, \mathrm{pH} \text {, REGION, } \\
\text { all interactions with REGION except } \\
\mathrm{LN}^{2} \text { (SOC):REGION, LN(TEB): REGION }\end{array}$ & 1.24 & $\begin{array}{l}\mathrm{LN}^{2}(\mathrm{SOC}), \mathrm{LN}(\mathrm{SOC}), \mathrm{C}: \mathrm{N}, \quad \mathrm{pH}^{2}, \mathrm{pH}, \\
\text { REGION, } \\
\text { all interactions with REGION except } \\
\mathrm{LN}^{2} \text { (SOC):REGION, } \\
\text { LN(SOC):REGION, }\end{array}$ & 1.24 \\
\hline \multirow[b]{2}{*}{$\begin{array}{l}\mathrm{LN}^{2} \text { (SOC), LN(SOC), C:N, CLAY min.soil, LN(TEB), } \\
\mathrm{pH}^{2} \text {, pH, REGION, } \\
\text { all interactions with REGION except } \\
\mathrm{LN}^{2} \text { (SOC):REGION, } \\
\text { LN(TEB):REGION, } \\
\text { CLAY }{ }_{\text {min.soil:REGION }}\end{array}$} & \multirow{2}{*}{1.24} & INGTER - REGION & \\
\hline & & $\begin{array}{l}\mathrm{LN}^{2}(\mathrm{SOC}), \mathrm{LN}(\mathrm{SOC}), \mathrm{C}: \mathrm{N}, \quad \mathrm{LN}(\mathrm{TEB}), \\
\mathrm{pH}^{2}, \mathrm{pH}, \mathrm{REGION}, \\
\text { all interactions with REGION except } \\
\mathrm{LN}^{2} \text { (SOC):REGION, } \\
\text { LN(SOC):REGION, }\end{array}$ & 1.24 \\
\hline \multirow{2}{*}{$\begin{array}{l}\mathrm{LN}^{2}(\mathrm{SOC}), \mathrm{LN}(\mathrm{SOC}), \mathrm{C}: \mathrm{N}, \quad \mathrm{LN}(\mathrm{TEB}), \mathrm{pH}^{2}, \mathrm{pH} \text {, } \\
\text { REGION, } \\
\text { all interactions with REGION except } \\
\text { LN2(SOC):REGION, } \\
\text { LN(TEB):REGION }\end{array}$} & \multirow[t]{2}{*}{1.23} & LN(TEB):REGION, pH²:REGION & \\
\hline & & $\begin{array}{l}\mathrm{LN}^{2}(\mathrm{SOC}), \mathrm{LN}(\mathrm{SOC}), \mathrm{C}: \mathrm{N}, \quad \mathrm{LN}(\mathrm{TEB}), \\
\mathrm{pH}^{2}, \mathrm{pH}, \mathrm{REGION}, \\
\text { all interactions with REGION except } \\
\text { LN'(SOC):REGION, }\end{array}$ & 1.25 \\
\hline $\begin{array}{l}\mathrm{LN}^{2}(\mathrm{SOC}), \mathrm{LN}(\mathrm{SOC}), \mathrm{C}: \mathrm{N}, \mathrm{LN}(\mathrm{TEB}), \mathrm{pH}^{2}, \mathrm{pH}, \\
\text { REGION, } \\
\text { all interactions with REGION except }\end{array}$ & 1.24 & $\begin{array}{l}\text { LN(SOC):REGION, } \\
\text { LN(TEB):REGION, } \\
\text { C:N:REGION }\end{array}$ & \\
\hline $\begin{array}{l}\text { LN2(SOC):REGION, } \\
\text { LN(TEB):REGION, } \mathrm{pH}^{2}: \text { REGION }\end{array}$ & & $\begin{array}{l}\text { LN(SOC), C:N, LN(TEB), } \mathrm{pH}^{2}, \mathrm{pH}, \\
\text { REGION, }\end{array}$ & 1.26 \\
\hline \multirow[t]{2}{*}{$\begin{array}{l}\mathrm{LN}^{2}(\mathrm{SOC}), \mathrm{LN}(\mathrm{SOC}), \mathrm{C}: \mathrm{N}, \mathrm{pH}^{2}, \mathrm{pH}, \mathrm{REGION}, \\
\text { all interactions with REGION except } \\
\mathrm{LN}^{2} \text { (SOC):REGION, } \mathrm{pH}^{2}: \text { REGION }\end{array}$} & 1.24 & $\begin{array}{l}\text { all interactions with REGION except } \\
\text { LN(SOC):REGION, } \\
\text { LN(SOC):REGION, } \\
\text { LN(TEB):REGION }\end{array}$ & \\
\hline & & all models including $\mathrm{LN}(\mathrm{N}$ stock) & $\geq 1.24$ \\
\hline
\end{tabular}


Table S8. Overall mean squared error (ms) after 10-fold cross-validation for the most relevant candidate model structures that explain variation in normalized productivity according to method 2 (actual/attainable mean annual increment - MAI) by soil variables (0-20 cm or mineral soil only) for Swedish spruce and pine forests. The selected model is marked in gray. Abbreviations: C: $\mathrm{N}=$ soil carbon to nitrogen ratio; $\mathrm{TEB}=$ total exchangeable bases; $\mathrm{pH}=\mathrm{pHKCl} ; \mathrm{CLAY}$ min.soil = clay fraction in the mineral soil; SOC $=$ soil organic carbon concentration. For soil C:N, data of depth $0-10 \mathrm{~cm}$ were used instead of $0-20 \mathrm{~cm}$ as the mean squared error of the single regression model was lowest in the former case (Table 2).

\begin{tabular}{|c|c|}
\hline Variables in model & Overall ms \\
\hline $\begin{array}{l}\text { LN }^{2} \text { (SOC), LN(SOC), LN(N stock), LN(C:N), SAND min.soil, CLAY min.soil, } \\
\text { LN(TEB), pH }\end{array}$ & 138 \\
\hline $\mathrm{LN}^{2}$ (SOC), LN(SOC), LN(N stock), LN(C:N), SAND min.soil, LN(TEB), pH & 138 \\
\hline$L N^{2}(S O C), L N(S O C), L N(N$ stock $), L N(C: N), S A N D_{\text {min.soil, }} p H$ & 138 \\
\hline $\mathrm{LN}^{2}(\mathrm{SOC}), \mathrm{LN}(\mathrm{SOC}), \mathrm{LN}(\mathrm{N}$ stock), $\mathrm{LN}(\mathrm{C}: \mathrm{N}), \mathrm{pH}$ & 139 \\
\hline $\mathrm{LN}^{2}(\mathrm{SOC}), \mathrm{LN}(\mathrm{SOC}), \mathrm{LN}(\mathrm{N}$ stock $), \mathrm{LN}(\mathrm{C}: \mathrm{N}), \mathrm{SAND}_{\text {min.soil }}$ & 143 \\
\hline
\end{tabular}

160 
Table S9. Tests of variable implementation in the IIASA-metric of constraints on nutrient availability, based on the complete Swedish database. Associations between residuals of normalized productivities in Fig. 9 and soil variables in the IIASA-metric are shown. For (near) significant variables (i.e. $P<0.10$ ), parameter estimates \pm s.e.m. and the proportion of variation explained $\left(R^{2}\right)$ are given. Abbreviations: $\mathrm{N}=$ north; $\mathrm{M}=$ middle; $\mathrm{S}=$ south; $\mathrm{SOC}=$ soil organic carbon concentration; $\mathrm{TEB}=$ total exchangeable bases; $\mathrm{pH} \mathrm{w}_{\mathrm{w}}=\mathrm{pH}$

185 measured in water; quad = parameter estimate for quadratic term; lin = parameter estimate for linear term of a quadratic function. Note that TEB is expressed here according to the standard definition (per kg dry weight as defined for the IIASA-metric), whereas elsewhere in this paper, TEB is referred to as a stock, i.e. an amount per $\mathrm{m}^{2}$ in the upper $20 \mathrm{~cm}$ of the soil, thus better representing the actual number of base cations available to plants. Error bars represent the s.e.m.

\begin{tabular}{|c|c|c|c|c|c|}
\hline Residuals of & Region & $\ln \mathrm{SOC}_{0-20 \mathrm{~cm}}[\%]$ & Sand $_{0-20 \mathrm{~cm}}[\%]$ & $\ln \mathrm{TEB}_{0-20 \mathrm{~cm}}\left[\mathrm{cmol}_{+} \mathrm{kg}^{-1}\right]$ & $\mathrm{pH}_{\mathrm{w}, 0-20 \mathrm{~cm}}$ \\
\hline \multirow[t]{3}{*}{$\begin{array}{l}\text { Residual MAI } \\
(\operatorname{method} 1)\end{array}$} & $\begin{array}{l}\mathrm{N} \\
(n=542)\end{array}$ & $\begin{array}{l}\text { slope }=-0.11 \pm 0.03 \\
P<0.01 \\
R^{2}=0.022\end{array}$ & $\begin{array}{l}\text { slope } \\
\quad=0.005 \pm 0.001 \\
P<0.01 \\
R^{2}=0.025\end{array}$ & $\begin{array}{l}\text { slope }=-0.04 \pm 0.02 \\
P=0.09 \\
R^{2}=0.003\end{array}$ & $\begin{array}{l}\text { quad }=-0.3 \pm 0.1 \\
\text { lin }=2.7 \pm 0.9 \\
P<0.01 \\
R^{2}=0.019\end{array}$ \\
\hline & $\begin{array}{l}M \\
(n=777)\end{array}$ & $\begin{array}{l}\text { slope }=-0.31 \pm 0.03 \\
P<0.01 \\
R^{2}=0.092\end{array}$ & $\begin{array}{l}\text { slope }=0.012 \pm 0.002 \\
P<0.01 \\
R^{2}=0.065\end{array}$ & $\begin{array}{l}\text { slope }=-0.20 \pm 0.03 \\
P<0.01 \\
R^{2}=0.052\end{array}$ & $\begin{array}{l}\text { quad }=-0.4 \pm 0.1 \\
\operatorname{lin}=4 \pm 1 \\
P<0.01 \\
R^{2}=0.055\end{array}$ \\
\hline & $\begin{array}{l}S \\
(n=946)\end{array}$ & $\begin{array}{l}\text { slope }=-0.56 \pm 0.04 \\
P<0.01 \\
R^{2}=0.141\end{array}$ & $\begin{array}{l}\text { slope }=0.015 \pm 0.002 \\
P<0.01 \\
R^{2}=0.076\end{array}$ & $\begin{array}{l}\text { slope }=-0.39 \pm 0.04 \\
P<0.01 \\
R^{2}=0.099\end{array}$ & $\begin{array}{l}\text { quad }=-0.86 \pm 0.07 \\
\text { lin }=8.4 \pm 0.6 \\
P<0.01 \\
R^{2}=0.166\end{array}$ \\
\hline $\begin{array}{l}\text { Actual/attainable } \\
\text { MAI } \\
(\text { method } 2)\end{array}$ & $\begin{array}{l}\text { entire } \\
\text { Sweden } \\
(n=955)\end{array}$ & $P=0.41$ & $P=0.33$ & $\begin{array}{l}\text { slope }=-0.6 \pm 0.4 \\
P=0.08 \\
R^{2}=0.002\end{array}$ & $P=0.73$ \\
\hline
\end{tabular}

190 
Table S10. Associations between single key soil variables and normalized productivity (method 1) for spruce and pine forests in southern Sweden. Significance $\left(P\right.$-values) of single soil variable effects on residual productivity (mean annual increment - MAI $\left[\mathrm{m}^{3}\right.$ $\mathrm{ha}^{-1} \mathrm{yr}^{-1}$ ) are given. For (near) significant variables (i.e. $P<0.10$ ), parameter estimates \pm s.e.m. and the proportion of variation explained $\left(R^{2}\right)$ are shown as well. Note that the results presented here deviate slightly from the data presented in Table 2, as separate regression analyses were performed for southern Sweden here instead of ANCOVA analyses with region as the factor, and results were based on a calibration subset here, instead of the complete database Moreover, $\mathrm{pH}_{\mathrm{w}}$ was used instead of $\mathrm{pH}_{\mathrm{KCl}}$ to keep the same variable for soil $\mathrm{pH}$ as in the original nutrient availability metric. Abbreviations: $\mathrm{S}=$ south; $\mathrm{SOC}=$ soil organic carbon concentration; Soil C:N = soil carbon to nitrogen ratio; quad = parameter estimate for quadratic term; lin = parameter estimate for linear term of a quadratic function.

\begin{tabular}{|c|c|c|c|c|c|}
\hline $\begin{array}{l}\text { Normalized } \\
\text { productivity } \\
\text { response }\end{array}$ & Region & $\begin{array}{l}\ln \mathrm{SOC}_{0-20 \mathrm{~cm}} \\
{[\%]}\end{array}$ & Soil C: $\mathbf{N}_{0-20 \mathrm{~cm}}$ & $\mathrm{pH}_{\mathrm{w}, 0-20 \mathrm{~cm}}$ & \\
\hline $\begin{array}{l}\text { Residual } \\
\text { MAI }\end{array}$ & $\begin{array}{l}\mathrm{S} \\
(n=473)\end{array}$ & $\begin{array}{l}\text { quad }=-0.18 \pm 0.06 \\
P<0.01 \\
\text { lin }=0.3 \pm 0.3 \\
P=0.22 \\
\text { intercept }=0.4 \pm 0.3 \\
P=0.19 \\
R_{\text {tot }}^{2}=0.134\end{array}$ & $\begin{array}{l}\text { slope }=-0.082 \pm 0.009 \\
P<0.01 \\
\text { intercept }=2.0 \pm 0.2 \\
P<0.01 \\
R^{2}=0.134\end{array}$ & $\begin{array}{l}\text { quad }=-0.9 \pm 0.1 \\
P<0.01 \\
\text { lin }=8.4 \pm 0.9 \\
P<0.01 \\
\text { intercept }=-19 \pm 2 \\
P<0.01 \\
R^{2} \text { tot }=0.172\end{array}$ & 215 \\
\hline
\end{tabular}


Table S11. Associations between single key soil variables and normalized productivity (method 2) for spruce forests in Sweden. Significance ( $P$-values) of single soil variable effects on actual/attainable productivity (mean annual increment $\left.-\mathrm{MAI}^{2} \mathrm{~m}^{3} \mathrm{ha}^{-1} \mathrm{yr}^{-1}\right]$ ) are given. For (near) significant variables (i.e. $P<0.10)$, parameter estimates \pm s.e.m. and the proportion of variation explained $\left(R^{2}\right)$ are shown as well. Note that the results presented here deviate slightly from the data presented in Table 2 , as they were based on a calibration subset here, instead of the complete database. Moreover, $\mathrm{pH}_{\mathrm{w}}$ was used instead of $\mathrm{pH}_{\mathrm{KCl}}$ to keep the same variable for soil $\mathrm{pH}$ as in the original nutrient availability metric. Abbreviations: $\mathrm{S}=$ south; $\mathrm{SOC}=$ soil organic carbon concentration; Soil C: $\mathrm{N}=$ soil carbon to nitrogen ratio; quad = parameter estimate for quadratic term; lin = parameter estimate for linear term of a quadratic function.

\begin{tabular}{llll}
\hline $\begin{array}{l}\text { Normalized } \\
\text { productivity } \\
\text { response }\end{array}$ & $\begin{array}{l}\text { In SOC } \mathbf{0 - 2 0 c m} \\
{[\%]}\end{array}$ & In soil C:N0-10cm & pH $_{\mathbf{w}, \mathbf{0 - 2 0 c m}}$ \\
\hline Actual/attainable & quad $=-2.8 \pm 0.6$ & slope $=-19 \pm 2$ & \\
MAI & $P<0.01$ & $P<0.01$ & slope $=2 \pm 1$ \\
$(n=475)$ & lin $=12 \pm 3$ & intercept $=102 \pm 7$ & $P=0.08$ \\
& $P<0.01$ & $P<0.01$ & intercept $=31 \pm 5$ \\
& intercept $=31 \pm 2$ & $R^{2}=0.138$ & $P<0.01$ \\
& $P<0.01$ & & $R^{2}=0.004$ \\
& $R^{2}=0.049$ & & \\
\hline
\end{tabular}


Table S12. Performance of adjusted metric 1 per soil moisture class. Significance $(P$-values) of metric-normalized productivity (mean annual increment - MAI $\left[\mathrm{m}^{3} \mathrm{ha}^{-1} \mathrm{yr}^{-1}\right]$ ) associations are given. For (near) significant relationships (i.e. $P<0.10$ ), parameter estimates \pm s.e.m. and the proportion of variation explained $\left(R^{2}\right)$ are shown as well. Abbreviations: $\mathrm{N}=$ north; $\mathrm{M}=$ middle; $\mathrm{S}=$ south.

\begin{tabular}{|c|c|c|c|c|c|}
\hline \multirow{2}{*}{$\begin{array}{l}\text { Normalized } \\
\text { productivity } \\
\text { response }\end{array}$} & \multirow[t]{2}{*}{ Region } & \multicolumn{4}{|l|}{$\begin{array}{l}\text { Adjusted metric } 1 \\
{\left[\mathrm{~m}^{3} \mathbf{h a}^{-1} \mathbf{y r}^{-1}\right]}\end{array}$} \\
\hline & & Dry & Fresh & Fresh-moist & Moist \\
\hline \multirow[t]{3}{*}{$\begin{array}{l}\text { Residual MAI } \\
\text { (method 1) }\end{array}$} & $\mathrm{N}$ & $\begin{array}{l}P=0.99 \\
n=10\end{array}$ & $\begin{array}{l}\text { slope }=0.5 \pm 0.1 \\
P<0.01 \\
R^{2}=0.071 \\
n=322\end{array}$ & $\begin{array}{l}\text { slope }=0.3 \pm 0.1 \\
P<0.01 \\
R^{2}=0.030 \\
n=201\end{array}$ & $\begin{array}{l}\text { slope }=1.0 \pm 0.1 \\
P=0.01 \\
R^{2}=0.539 \\
n=8\end{array}$ \\
\hline & M & $\begin{array}{l}P=0.78 \\
n=16\end{array}$ & $\begin{array}{l}\text { slope }=0.8 \pm 0.1 \\
P<0.01 \\
R^{2}=0.089 \\
n=488\end{array}$ & $\begin{array}{l}\text { slope }=0.9 \pm 0.1 \\
P<0.01 \\
R^{2}=0.135 \\
n=262\end{array}$ & $\begin{array}{l}P=0.45 \\
n=10\end{array}$ \\
\hline & S & $\begin{array}{l}P=0.51 \\
n=6\end{array}$ & $\begin{array}{l}\text { slope }=1.4 \pm 0.2 \\
P<0.01 \\
R^{2}=0.089 \\
n=488\end{array}$ & $\begin{array}{l}\text { slope }=1.7 \pm 0.2 \\
P<0.01 \\
R^{2}=0.258 \\
n=183\end{array}$ & $\begin{array}{l}P=0.95 \\
n=8\end{array}$ \\
\hline $\begin{array}{l}\text { Actual/attainable } \\
\text { MAI } \\
\text { (method 2) }\end{array}$ & $\begin{array}{l}\text { entire } \\
\text { Sweden }\end{array}$ & $\begin{array}{l}P=0.20 \\
n=19\end{array}$ & $\begin{array}{l}\text { slope }=3 \pm 2 \\
P=0.08 \\
R^{2}=0.003 \\
n=640\end{array}$ & $\begin{array}{l}\text { slope }=4 \pm 2 \\
P=0.02 \\
R^{2}=0.017 \\
n=285\end{array}$ & $\begin{array}{l}P=0.67 \\
n=10\end{array}$ \\
\hline
\end{tabular}


Table S13. Performance of adjusted metric 1 per soil type. Significance ( $P$-values) of metric-normalized productivity (mean annual increment - MAI $\left[\mathrm{m}^{3} \mathrm{ha}^{-1} \mathrm{yr}^{-1}\right]$ ) associations are given. For (near) significant relationships (i.e. $P<0.10$ ), parameter estimates \pm s.e.m. and the proportion of variation explained $\left(R^{2}\right)$ are shown as well. Abbreviations: $\mathrm{N}=$ north; $\mathrm{M}=$ middle; $\mathrm{S}=$ south.

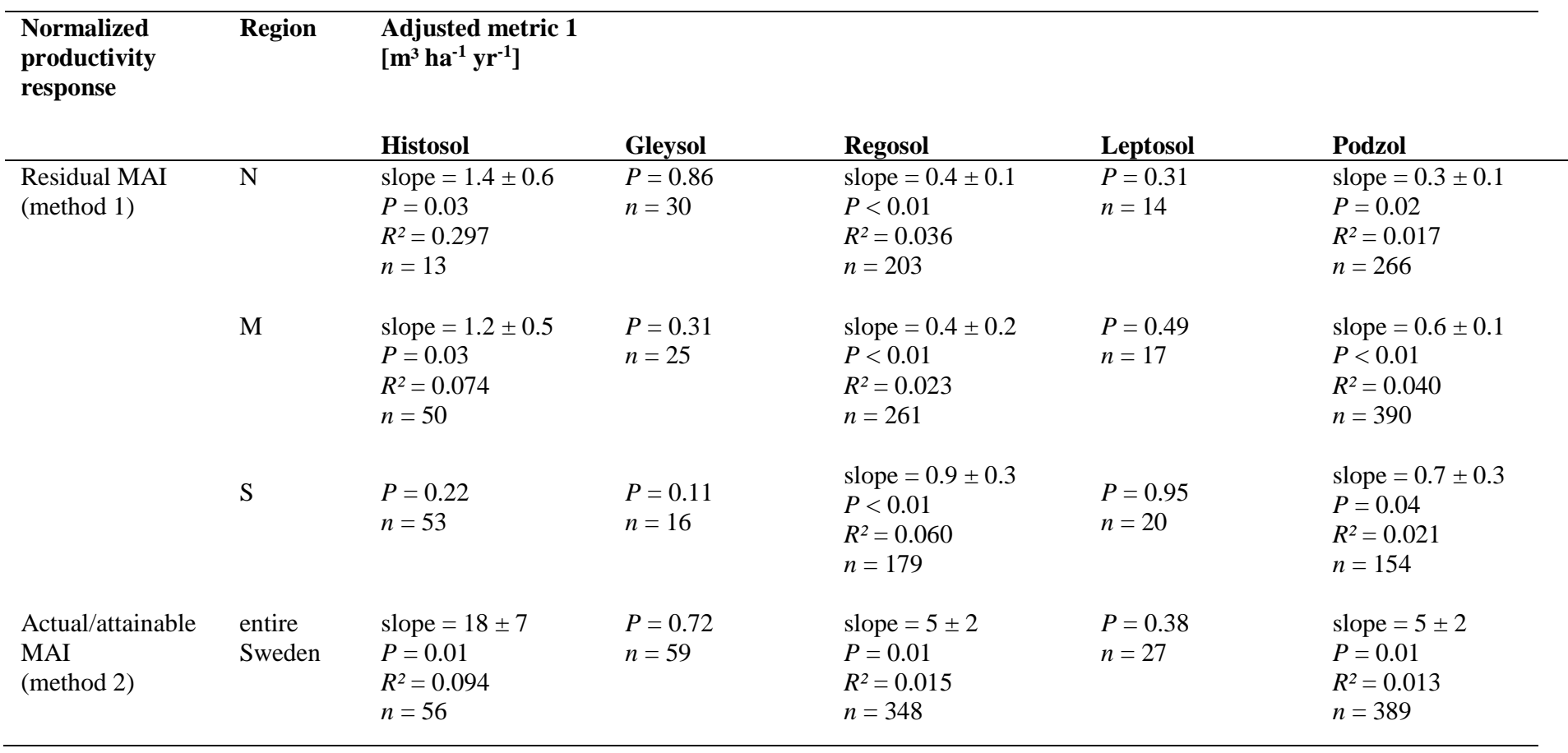


Table S14. Performance of adjusted metric 2 per soil moisture class. Significance $(P$-values) of metric-normalized productivity (mean annual increment - MAI $\left[\mathrm{m}^{3} \mathrm{ha}^{-1} \mathrm{yr}^{-1}\right]$ ) associations are given. For (near) significant relationships (i.e. $P<0.10$ ), parameter estimates \pm s.e.m. and the proportion of variation explained $\left(R^{2}\right)$ are shown as well. Abbreviations: $\mathrm{N}=$ north; $\mathrm{M}=$ middle; $\mathrm{S}=$ south.

\begin{tabular}{|c|c|c|c|c|c|}
\hline \multirow{2}{*}{$\begin{array}{l}\text { Normalized } \\
\text { productivity } \\
\text { response }\end{array}$} & \multirow[t]{2}{*}{ Region } & \multicolumn{4}{|l|}{$\begin{array}{l}\text { Adjusted metric } 2 \\
{[\%]}\end{array}$} \\
\hline & & Dry & Fresh & Fresh-moist & Moist \\
\hline \multirow[t]{3}{*}{$\begin{array}{l}\text { Residual MAI } \\
\text { (method 1) }\end{array}$} & $\mathrm{N}$ & $\begin{array}{l}\text { slope }=0.07 \pm 0.03 \\
P=0.02 \\
R^{2}=0.148 \\
n=32\end{array}$ & $\begin{array}{l}\text { slope }=0.07 \pm 0.01 \\
P<0.01 \\
R^{2}=0.055 \\
n=383\end{array}$ & $\begin{array}{l}\text { slope }=0.14 \pm 0.02 \\
P<0.01 \\
R^{2}=0.240 \\
n=122\end{array}$ & $\begin{array}{l}P=0.26 \\
n=4\end{array}$ \\
\hline & M & $\begin{array}{l}P=0.39 \\
n=47\end{array}$ & $\begin{array}{l}\text { slope }=0.10 \pm 0.02 \\
P<0.01 \\
R^{2}=0.042 \\
n=514\end{array}$ & $\begin{array}{l}\text { slope }=0.18 \pm 0.03 \\
P<0.01 \\
R^{2}=0.141 \\
n=208\end{array}$ & $\begin{array}{l}P=0.33 \\
n=7\end{array}$ \\
\hline & $\mathrm{S}$ & $\begin{array}{l}P=0.95 \\
n=44\end{array}$ & $\begin{array}{l}\text { slope }=0.11 \pm 0.03 \\
P<0.01 \\
R^{2}=0.026 \\
n=669\end{array}$ & $\begin{array}{l}\text { slope }=0.27 \pm 0.04 \\
P<0.01 \\
R^{2}=0.211 \\
n=216\end{array}$ & $\begin{array}{l}\text { slope }=0.5 \pm 0.2 \\
P=0.02 \\
R^{2}=0.233 \\
n=17\end{array}$ \\
\hline $\begin{array}{l}\text { Actual/attainable } \\
\text { MAI } \\
(\operatorname{method} 2)\end{array}$ & $\begin{array}{l}\text { entire } \\
\text { Sweden }\end{array}$ & $\begin{array}{l}\text { slope }=5 \pm 2 \\
P=0.03 \\
R^{2}=0.334 \\
n=11\end{array}$ & $\begin{array}{l}\text { slope }=2.7 \pm 0.3 \\
P<0.01 \\
R^{2}=0.167 \\
n=320\end{array}$ & $\begin{array}{l}\text { slope }=2.0 \pm 0.4 \\
P<0.01 \\
R^{2}=0.132 \\
n=135\end{array}$ & $\begin{array}{l}P=0.92 \\
n=6\end{array}$ \\
\hline
\end{tabular}


Table S15. Performance of adjusted metric 2 per soil type. Significance ( $P$-values) of metric-normalized productivity (mean annual increment - MAI $\left[\mathrm{m}^{3} \mathrm{ha}^{-1} \mathrm{yr}^{-1}\right]$ ) associations are given. For (near) significant relationships (i.e. $P<0.10$ ), parameter estimates \pm s.e.m. and the proportion of variation explained $\left(R^{2}\right)$ are shown as well. Abbreviations: $\mathrm{N}=$ north; $\mathrm{M}=$ middle; $\mathrm{S}=$ south.

\begin{tabular}{|c|c|c|c|c|c|c|}
\hline \multirow{2}{*}{$\begin{array}{l}\text { Normalized } \\
\text { productivity } \\
\text { response }\end{array}$} & \multirow[t]{2}{*}{ Region } & \multicolumn{5}{|l|}{$\begin{array}{l}\text { Adjusted metric } 2 \\
{[\%]}\end{array}$} \\
\hline & & Histosol & Gleysol & Regosol & Leptosol & Podzol \\
\hline \multirow[t]{3}{*}{$\begin{array}{l}\text { Residual MAI } \\
\text { (method 1) }\end{array}$} & $\mathrm{N}$ & $\begin{array}{l}\text { slope }=0.23 \pm 0.08 \\
P=0.01 \\
R^{2}=0.381 \\
n=13\end{array}$ & $\begin{array}{l}\text { slope } \\
\quad=0.08 \pm 0.04 \\
P=0.06 \\
R^{2}=0.091 \\
n=30\end{array}$ & $\begin{array}{l}\text { slope }=0.07 \pm 0.02 \\
P<0.01 \\
R^{2}=0.064 \\
n=203\end{array}$ & $\begin{array}{l}\text { slope }=0.16 \pm 0.05 \\
P<0.01 \\
R^{2}=0.370 \\
n=14\end{array}$ & $\begin{array}{l}\text { slope } \\
\quad=0.07 \pm 0.02 \\
P<0.01 \\
R^{2}=0.051 \\
n=266\end{array}$ \\
\hline & M & $\begin{array}{l}\text { slope }=0.19 \pm 0.07 \\
P=0.01 \\
R^{2}=0.098 \\
n=50\end{array}$ & $\begin{array}{l}\text { slope }=0.2 \pm 0.1 \\
P=0.10 \\
R^{2}=0.072 \\
n=25\end{array}$ & $\begin{array}{l}\text { slope }=0.08 \pm 0.03 \\
P<0.01 \\
R^{2}=0.027 \\
n=261\end{array}$ & $\begin{array}{l}P=0.99 \\
n=17\end{array}$ & $\begin{array}{l}\text { slope } \\
\quad=0.13 \pm 0.03 \\
P<0.01 \\
R^{2}=0.059 \\
n=390\end{array}$ \\
\hline & S & $\begin{array}{l}\text { slope }=0.36 \pm 0.08 \\
P<0.01 \\
R^{2}=0.172 \\
n=93\end{array}$ & $\begin{array}{l}P=0.23 \\
n=34\end{array}$ & $\begin{array}{l}\text { slope }=0.12 \pm 0.03 \\
P<0.01 \\
R^{2}=0.032 \\
n=358\end{array}$ & $\begin{array}{l}P=0.79 \\
n=40\end{array}$ & $\begin{array}{l}\text { slope } \\
\quad=0.14 \pm 0.04 \\
P<0.01 \\
R^{2}=0.036 \\
n=322\end{array}$ \\
\hline $\begin{array}{l}\text { Actual/attainable } \\
\text { MAI } \\
(\text { method } 2)\end{array}$ & $\begin{array}{l}\text { entire } \\
\text { Sweden }\end{array}$ & $\begin{array}{l}P=0.40 \\
n=25\end{array}$ & $\begin{array}{l}\text { slope }=1.9 \pm 0.8 \\
P=0.02 \\
R^{2}=0.141 \\
n=30\end{array}$ & $\begin{array}{l}\text { slope }=3.3 \pm 0.5 \\
P<0.01 \\
R^{2}=0.232 \\
n=170\end{array}$ & $\begin{array}{l}\text { slope }=2.0 \pm 0.9 \\
P=0.04 \\
R^{2}=0.205 \\
n=15\end{array}$ & $\begin{array}{l}\text { slope }=2.5 \pm 0.5 \\
P<0.01 \\
R^{2}=0.133 \\
n=199\end{array}$ \\
\hline
\end{tabular}


Table S16. Tests of variable implementation in adjusted nutrient availability metric 1, based on the complete Swedish database. Associations between residuals of normalized productivities in Fig. 10 and soil variables in adjusted metric 2 are shown. For (near) significant variables (i.e. $P<0.10)$, parameter estimates \pm s.e.m. and the proportion of variation explained $\left(R^{2}\right)$ are given. Abbreviations: $\mathrm{N}$ $=$ north $; \mathrm{M}=$ middle; $\mathrm{S}=$ south $\mathrm{SOC}=$ soil organic carbon concentration; Soil $\mathrm{C}: \mathrm{N}=$ soil carbon to nitrogen ratio; $\mathrm{pH}=\mathrm{pH}$ measured in water. Error bars represent the s.e.m.

\begin{tabular}{|c|c|c|c|c|}
\hline & Region & $\ln \mathrm{SOC}_{0-20 \mathrm{~cm}}[\%]$ & Soil C:N0-20cm & $\mathrm{pH}_{\mathrm{w}, 0-20 \mathrm{~cm}}$ \\
\hline \multirow[t]{3}{*}{$\begin{array}{l}\text { Residual MAI } \\
\text { (method 1) }\end{array}$} & $\begin{array}{l}\mathrm{N} \\
(n=542)\end{array}$ & $P=0.40$ & $P=0.24$ & $P=0.28$ \\
\hline & $\begin{array}{l}\mathrm{M} \\
(n=777)\end{array}$ & $\begin{array}{l}\text { slope }=-0.06 \pm 0.03 \\
P=0.08 \\
R^{2}=0.003\end{array}$ & $\begin{array}{l}\text { slope }=0.011 \pm 0.005 \\
P=0.03 \\
R^{2}=0.005\end{array}$ & $P=0.14$ \\
\hline & $\begin{array}{l}S \\
(n=473)\end{array}$ & $P=0.36$ & $P=0.73$ & $P=0.28$ \\
\hline $\begin{array}{l}\text { Actual/attainable MAI } \\
(\operatorname{method} 2)\end{array}$ & $\begin{array}{l}\text { entire Sweden } \\
(n=955)\end{array}$ & $\begin{array}{l}\text { slope }=1.7 \pm 0.4 \\
P<0.01 \\
R^{2}=0.016\end{array}$ & $\begin{array}{l}\text { slope }=-0.33 \pm 0.07 \\
P<0.01 \\
R^{2}=0.021\end{array}$ & $P=0.17$ \\
\hline
\end{tabular}


Table S17. Tests of variable implementation in adjusted nutrient availability metric 2, based on the complete Swedish database. Associations between residuals of normalized productivities in Fig. 11 and soil variables in adjusted metric 2 are shown. For (near) significant variables (i.e. $P<0.10$ ), parameter estimates \pm s.e.m. and the proportion of variation explained $\left(R^{2}\right)$ are given. Abbreviations: $\mathrm{N}$ $=$ north $; \mathrm{M}=$ middle $; \mathrm{S}=$ south SOC = soil organic carbon concentration; Soil $\mathrm{C}: \mathrm{N}=$ soil carbon to nitrogen ratio; $\mathrm{pH}=\mathrm{pH}$ measured in 290 water. Error bars represent the s.e.m.

\begin{tabular}{|c|c|c|c|c|}
\hline Residuals of & Region & $\ln \mathrm{SOC}_{0-20 \mathrm{~cm}}[\%]$ & In soil C: $N_{0-10 \mathrm{~cm}}$ & $\mathrm{pH}_{\mathrm{w}, 0-20 \mathrm{~cm}}$ \\
\hline \multirow[t]{3}{*}{$\begin{array}{l}\text { Residual MAI } \\
\text { (method 1) }\end{array}$} & $\begin{array}{l}\mathrm{N} \\
(n=545)\end{array}$ & $\begin{array}{l}\text { slope }=-0.11 \pm 0.03 \\
P<0.01 \\
R^{2}=0.023\end{array}$ & $P=0.55$ & $\begin{array}{l}\text { slope }=0.16 \pm 0.07 \\
P=0.02 \\
R^{2}=0.008\end{array}$ \\
\hline & $\begin{array}{l}\text { M } \\
(n=780)\end{array}$ & $\begin{array}{l}\text { slope }=-0.30 \pm 0.03 \\
P<0.01 \\
R^{2}=0.093\end{array}$ & $P=0.94$ & $\begin{array}{l}\text { slope }=0.37 \pm 0.09 \\
P=0.02 \\
R^{2}=0.020\end{array}$ \\
\hline & $\begin{array}{l}\mathrm{S} \\
(n=950)\end{array}$ & $\begin{array}{l}\text { slope }=-0.37 \pm 0.04 \\
P<0.01 \\
R^{2}=0.067\end{array}$ & $P=0.16$ & $P=0.60$ \\
\hline $\begin{array}{l}\text { Actual/attainable MAI } \\
(\operatorname{method} 2)\end{array}$ & $\begin{array}{l}\text { entire Sweden } \\
(n=475)\end{array}$ & $\begin{array}{l}\text { slope }=1.0 \pm 0.6 \\
P=0.09 \\
R^{2}=0.004\end{array}$ & $P=0.34$ & $\begin{array}{l}\text { slope }=-5 \pm 1 \\
P<0.01 \\
R^{2}=0.036\end{array}$ \\
\hline
\end{tabular}


Table S18. Tests of variable implementation in adjusted nutrient availability metric 1, based on selected nutrient availability gradients in Sweden. Associations between residuals of normalized productivities in Table 4 and soil variables in adjusted metric 1 are shown. For 295 (near) significant variables (i.e. $P<0.10)$, parameter estimates \pm s.e.m. and the proportion of variation explained $\left(R^{2}\right)$ are given. For Norway spruce, no TEB gradient without substantial variation in climate was found, so that only for Scots pine, there was a gradient in TEB. Abbreviations: $\mathrm{SOC}=$ soil organic carbon concentration; Soil C:N = soil carbon to nitrogen ratio; $\mathrm{pH}_{\mathrm{w}}=\mathrm{pH}$ measured in water; $\mathrm{TEB}$ $=$ total exchangeable bases. Error bars represent the s.e.m.

\begin{tabular}{|c|c|c|c|c|}
\hline $\begin{array}{l}\text { Dominant tree } \\
\text { species }\end{array}$ & Gradient type & $\ln \mathrm{SOC}_{0-20 \mathrm{~cm}}[\%]$ & Soil C:No-20cm & $\mathrm{pH}_{\mathrm{w}, 0-20 \mathrm{~cm}}$ \\
\hline \multirow[t]{2}{*}{ Norway spruce } & $\begin{array}{l}\text { Soil moisture } \\
(n=132)\end{array}$ & $P=0.80$ & $P=0.38$ & $P=0.89$ \\
\hline & $\begin{array}{l}\text { Productivity } \\
(n=78)\end{array}$ & $P=0.62$ & $\begin{array}{l}\text { slope }=-0.06 \pm 0.03 \\
P=0.06 \\
R^{2}=0.033\end{array}$ & $\begin{array}{l}\text { slope }=1.0 \pm 0.4 \\
P<0.01 \\
R^{2}=0.083\end{array}$ \\
\hline \multirow[t]{3}{*}{ Scots pine } & $\begin{array}{l}\text { Soil moisture } \\
(n=141)\end{array}$ & $P=0.83$ & $P=0.62$ & $P=0.79$ \\
\hline & $\begin{array}{l}\text { TEB } \\
(n=59)\end{array}$ & $P=0.35$ & $P=0.70$ & $P=0.22$ \\
\hline & $\begin{array}{l}\text { Productivity } \\
(n=67)\end{array}$ & $P=0.94$ & $P=0.50$ & $P=0.84$ \\
\hline
\end{tabular}


Table S19. Tests of variable implementation in adjusted nutrient availability metric 2, based on selected nutrient availability gradients in Sweden. Associations between residuals of productivities in Table 5 and soil variables in adjusted metric 2 are shown. For (near) 305 significant variables (i.e. $P<0.10$ ), parameter estimates \pm s.e.m. and the proportion of variation explained $\left(R^{2}\right)$ are given. For Norway spruce, no TEB gradient without substantial variation in climate was found, so that only for Scots pine, there was a gradient in TEB. Abbreviations: $\mathrm{SOC}=$ soil organic carbon concentration; Soil C:N = soil carbon to nitrogen ratio; $\mathrm{pH}=\mathrm{pH}$ measured in water; TEB $=$ total exchangeable bases. Error bars represent the s.e.m.

\begin{tabular}{|c|c|c|c|c|}
\hline $\begin{array}{l}\text { Dominant tree } \\
\text { species }\end{array}$ & Gradient type & $\ln \mathrm{SOC}_{0-20 \mathrm{~cm}}[\%]$ & In soil C:No-10cm & $\mathrm{pH}_{\mathrm{w}, 0-20 \mathrm{~cm}}$ \\
\hline \multirow[t]{2}{*}{ Norway spruce } & $\begin{array}{l}\text { Soil moisture } \\
(n=132)\end{array}$ & $\begin{array}{l}\text { slope }=-0.3 \pm 0.1 \\
P=0.02 \\
R^{2}=0.031\end{array}$ & $P=0.96$ & $P=0.88$ \\
\hline & $\begin{array}{l}\text { Productivity } \\
(n=78)\end{array}$ & $\begin{array}{l}\text { slope }=-0.5 \pm 0.2 \\
P<0.01 \\
R^{2}=0.111\end{array}$ & $\begin{array}{l}\text { slope }=-1.9 \pm 0.7 \\
P=0.01 \\
R^{2}=0.065\end{array}$ & $\begin{array}{l}\text { slope }=0.6 \pm 0.4 \\
P=0.09 \\
R^{2}=0.025\end{array}$ \\
\hline \multirow[t]{3}{*}{ Scots pine } & $\begin{array}{l}\text { Soil moisture } \\
(n=141)\end{array}$ & $\begin{array}{l}\text { slope }=-0.37 \pm 0.09 \\
P<0.01 \\
R^{2}=0.098\end{array}$ & $P=0.77$ & $\begin{array}{l}\text { slope }=0.6 \pm 0.3 \\
P=0.03 \\
R^{2}=0.026\end{array}$ \\
\hline & $\begin{array}{l}\text { TEB } \\
(n=59)\end{array}$ & $\begin{array}{l}\text { slope }=-0.3 \pm 0.1 \\
P=0.03 \\
R^{2}=0.065\end{array}$ & $P=0.86$ & $P=0.58$ \\
\hline & $\begin{array}{l}\text { Productivity } \\
(n=67)\end{array}$ & $P=0.31$ & $P=0.78$ & $P=0.71$ \\
\hline
\end{tabular}




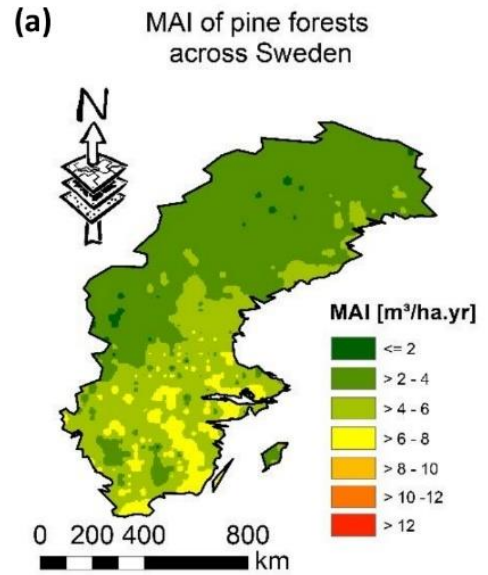

(c) Attainable MAI of spruce forests across Sweden

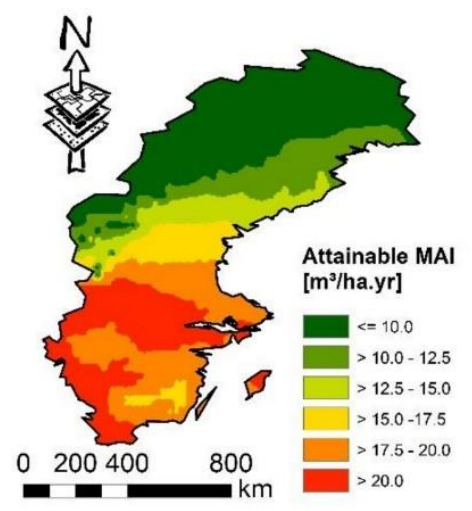

(b) MAl of spruce forests

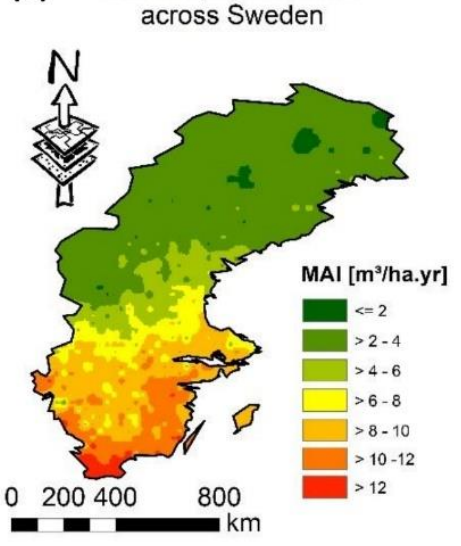

(d) Soil C: $\mathrm{N}$ ratios $(0-10 \mathrm{~cm})$ in Swedish conifer forests

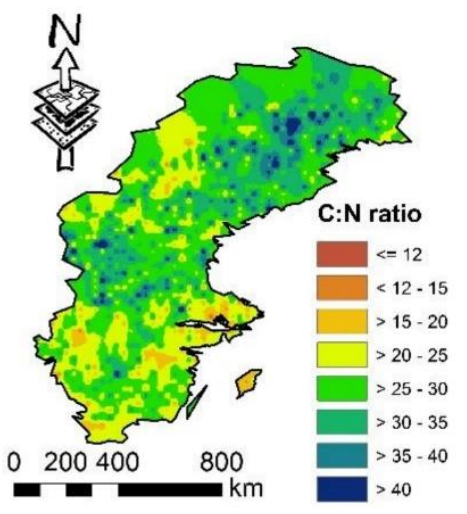

Figure S1. Productivities (mean annual increment - MAI) of (a) pine and (b) spruce, (c) attainable productivity for spruce (Bergh et al., 315 2005) and (d) soil carbon to nitrogen $(\mathrm{C}: \mathrm{N})$ ratio of conifer forests in Sweden. 


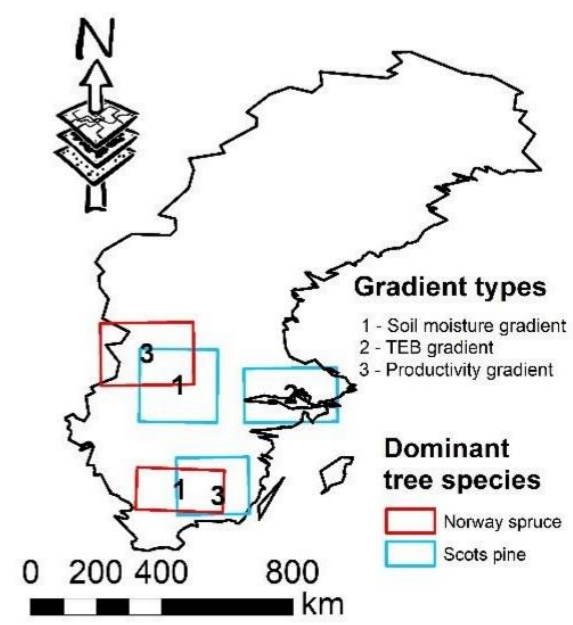

Figure S2. Overview of the selected nutrient availability gradients in Sweden. Gradients were selected by manually searching for regions 330 with high spatial variation in either soil moisture, total exchangeable bases (TEB) or productivity for spruce and pine forests, using the ArcGIS software (ESRI, 2011). For Norway spruce, no TEB gradient without substantial variation in climate was found, so that only for Scots pine, there was a gradient in TEB. Each gradient consisted of $\geq 40$ data points, and thus represented a sub-selection of the complete Swedish database. 
(a)
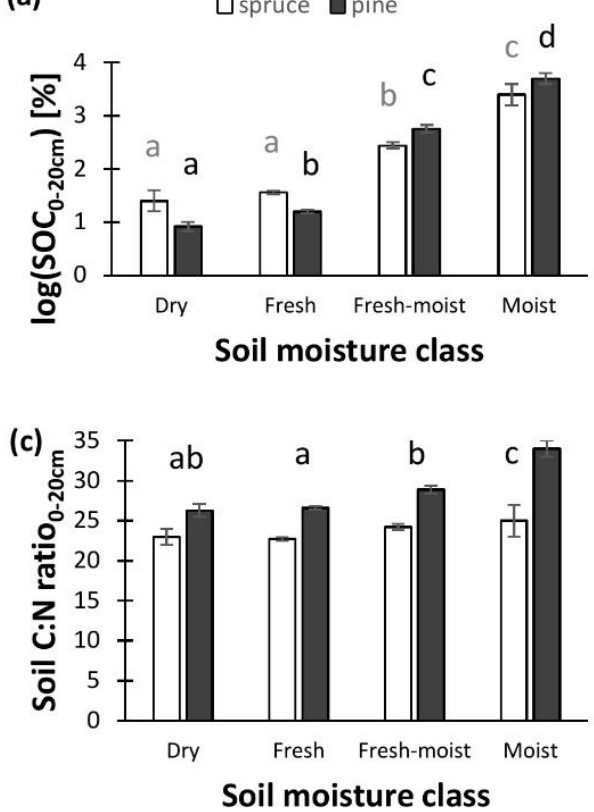

(e)
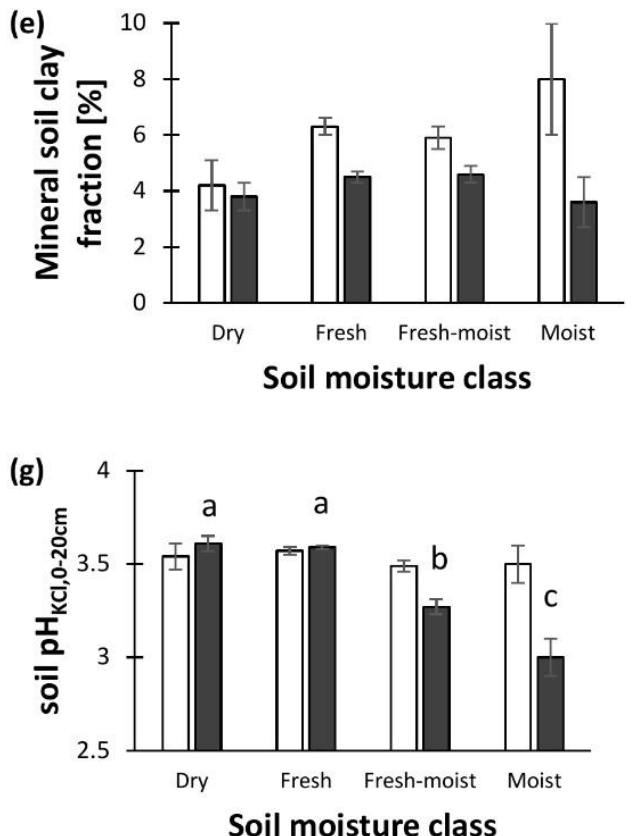

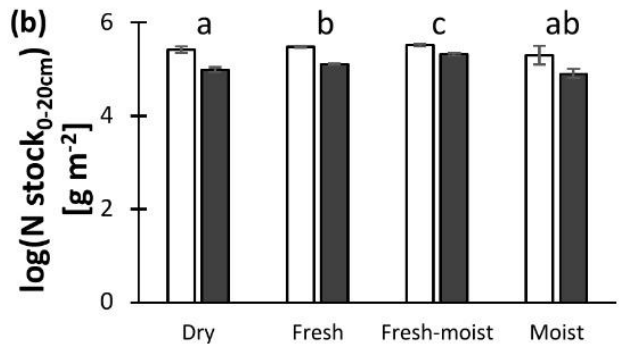

Soil moisture class
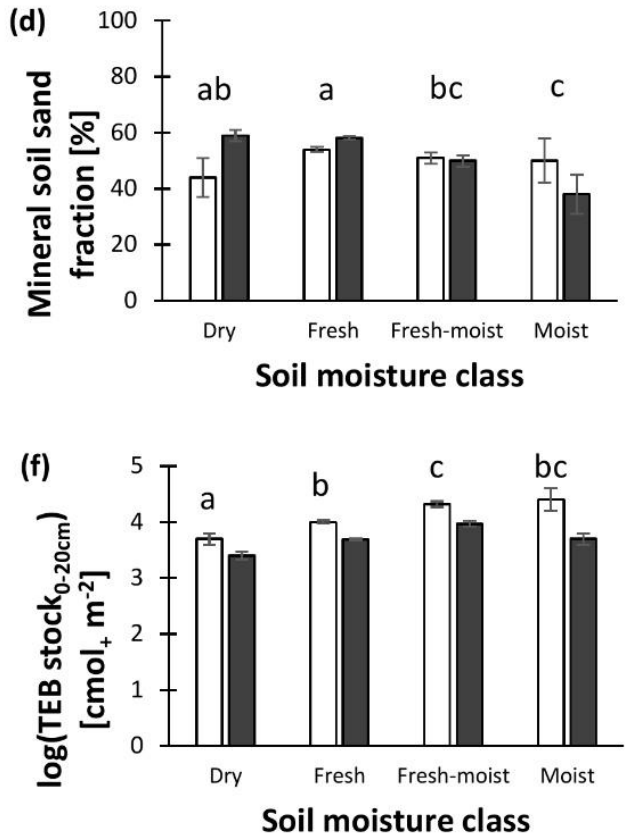

Figure S3. Soil conditions in spruce and pine forests with varying soil moisture. Abbreviations: SOC $=$ soil organic carbon concentration; soil $\mathrm{C}: \mathrm{N}$ ratio $=$ soil carbon to nitrogen ratio; $\mathrm{TEB}=$ total exchangeable bases. Letters indicate statistical differences among moisture classes, either within spruce and pine forests (if the moisture $\mathrm{x}$ species interaction was included in the model after cross-validation) or for spruce and pine forests combined (if the moisture $\mathrm{x}$ species interaction was not included in the final model). Error bars represent the s.e.m. 
(a)
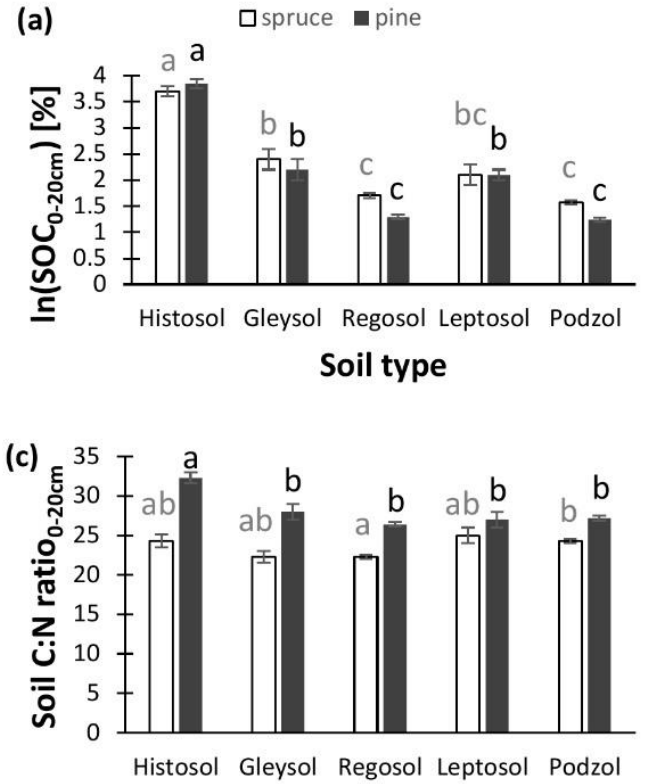

Soil type

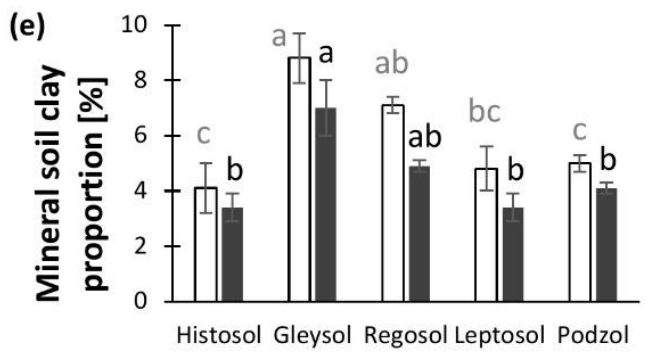

Soil type

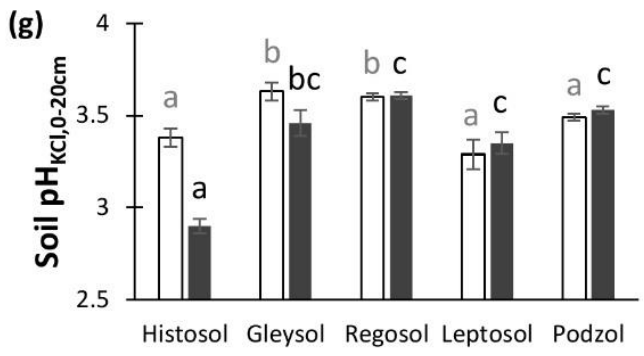

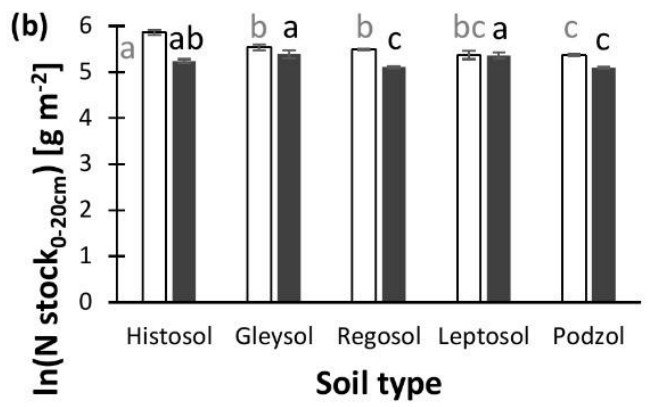

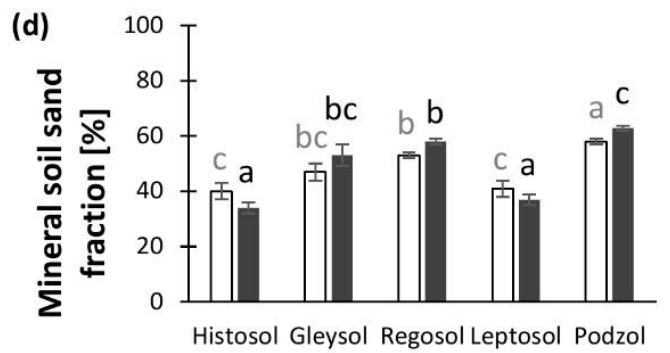

Soil type

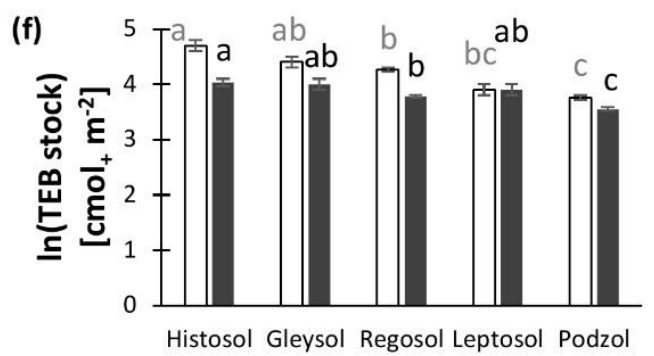

Soil type

Soil type

Figure S4. Soil conditions in spruce and pine forests for the most common soil types in Sweden. Abbreviations: SOC = soil organic carbon concentration; soil $\mathrm{C}: \mathrm{N}$ ratio $=$ soil carbon to nitrogen ratio; TEB $=$ total exchangeable bases. Letters indicate statistical differences among soil types, for each panel within spruce and pine forests (the moisture $\mathrm{x}$ species interaction was included in each model after crossvalidation). Error bars represent the s.e.m. 

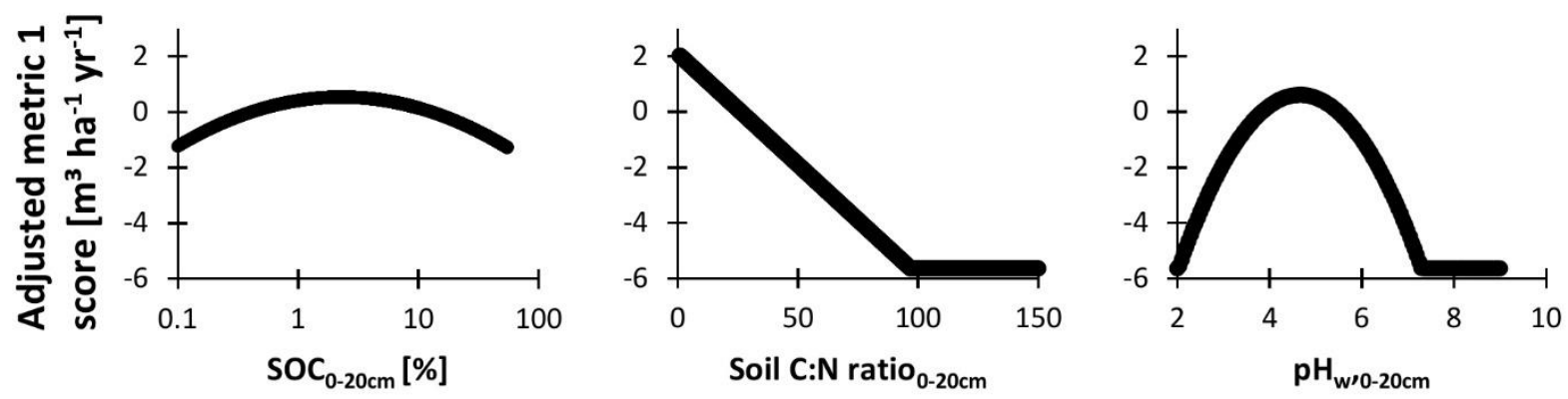

350 Figure S5. Adjusted soil scores in nutrient availability metric 1 for soil organic carbon concentration (SOC), soil carbon to nitrogen (C:N) ratio and $\mathrm{pH}$ measured in water $\left(\mathrm{pH}_{\mathrm{w}}\right)$. Curves were drawn based on regression equations from Table $\mathrm{S} 10$. Minimum constraints were added to avoid artefacts if applied to other databases. 

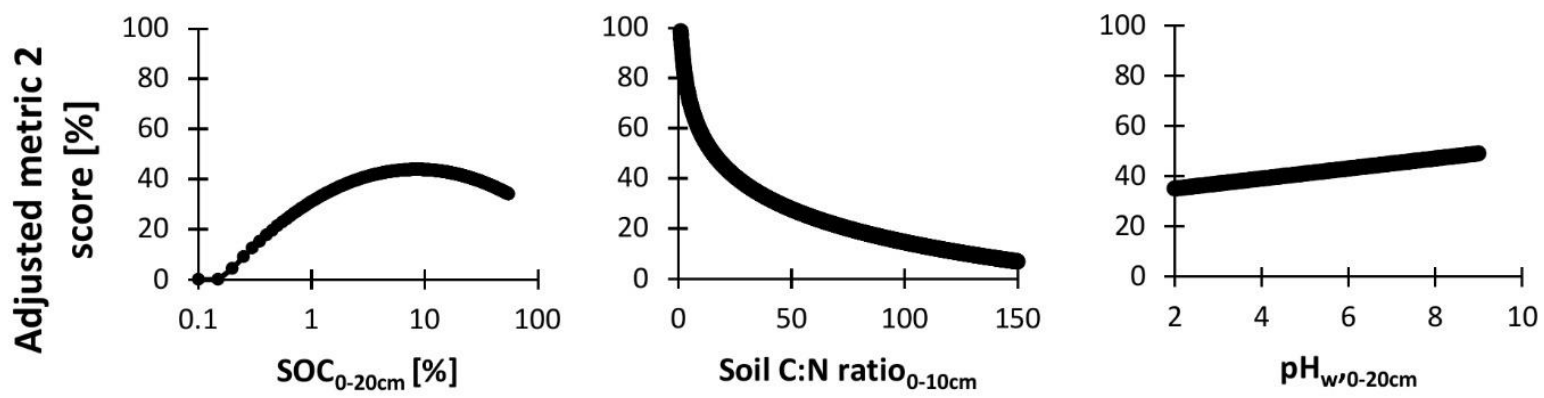

Figure S6. Adjusted soil scores in nutrient availability metric 2 for soil organic carbon concentration (SOC), soil carbon to nitrogen (C:N) ratio and $\mathrm{pH}$ measured in water $\left(\mathrm{pH}_{\mathrm{w}}\right)$. Curves were drawn based on regression equations from Table S11. Minimum constraints were 380 added to avoid artefacts if applied to other databases. 